\title{
Particle-hole symmetric localization in two dimensions
}

\author{
Olexei Motrunich, ${ }^{1}$ Kedar Damle, ${ }^{2}$ and David A. Huse ${ }^{1}$ \\ ${ }^{1}$ Physics Department, Princeton University, Princeton, New Jersey 08544 \\ ${ }^{2}$ Physics Department, Harvard University, Cambridge, Massachusetts 02138
}

(Received 5 August 2001; published 23 January 2002)

\begin{abstract}
We revisit two-dimensional particle-hole symmetric sublattice localization problem, focusing on the origin of the observed singularities in the density of states $\rho(E)$ at the band center $E=0$. The most general system of this kind [R. Gade, Nucl. Phys. B 398, 499 (1993)] exhibits critical behavior and has $\rho(E)$ that diverges stronger than any integrable power law, while the special random vector potential model of Ludwig et al. [Phys. Rev. B 50, 7526 (1994)] has instead a power-law density of states with a continuously varying dynamical exponent. We show that the latter model undergoes a dynamical transition with increasing disorder-this transition is a counterpart of the static transition known to occur in this system; in the strong-disorder regime, we identify the low-energy states of this model with the local extrema of the defining two-dimensional Gaussian random surface. Furthermore, combining this "surface fluctuation" mechanism with a renormalization group treatment of a related vortex glass problem leads us to argue that the asymptotic low- $E$ behavior of the density of states in the general case is $\rho(E) \sim E^{-1} e^{-c|\ln E|^{2 / 3}}$, different from earlier prediction of Gade. We also study the localized phases of such particle-hole symmetric systems and identify a Griffiths "string" mechanism that generates singular power-law contributions to the low-energy density of states in this case.
\end{abstract}

DOI: 10.1103/PhysRevB.65.064206

PACS number(s): 72.90.+y, 74.40.+k

\section{INTRODUCTION}

This paper is devoted to a careful study of the localization properties of certain two-dimensional systems with some special symmetry properties that make them stand apart from the other more generic universality classes of Anderson localization in two dimensions. The models we consider are simply tight-binding models of noninteracting particles moving on bipartite lattices in two dimensions (2D). ${ }^{1}$ The hopping matrix elements are random, but there are no on-site potentials, so the spectrum is strictly particle-hole symmetric. Such 2D localization problems have received particular attention $^{2-4}$ in the context of the integer quantum Hall plateau transition studies, and exhibit critical delocalized states only at the special energy $E=0$. At all nonzero energies the eigenstates are exponentially localized with a finite localization length.

Unlike the quantum Hall systems, which have an analytic density of states (DOS) at the corresponding delocalized critical points in the spectrum, the bipartite random hopping (BPRH) models have a strongly singular DOS at the band center. This unusual feature makes the sublattice problems very interesting in their own right since the disorder evidently has a very dramatic effect in the presence of the particle-hole symmetry.

Recent studies of the quasiparticle localization in dirty superconductors, where a somewhat analogous particle-hole symmetry appears naturally in the Bogoliubov-de Gennes formulation, have resulted in renewed interest in such special localization problems. For instance, the case with broken spin-rotation invariance ${ }^{5}$ can be formulated as a pure imaginary random hopping (ImRH) problem, and such ImRH problems constitute the simplest particle-hole symmetric generalization of the real BPRH models, obtained from the corresponding real BPRH by allowing additional purely imaginary hopping amplitudes between the sites of the same sublattice and performing a gauge transformation on one sublattice to make the intersublattice couplings imaginary. In the generic case, all such systems show some enhancement in the quasiparticle density of states at the band center, while the special case of a spinless superconductor with time reversal invariance (which constrains the couplings within a sublattice to be zero) maps precisely onto a real bipartite localization problem with all the concomitant strong spectral singularities.

To put our analysis of the bipartite models in context, it is useful to begin with a quick summary of what is known in the literature: Ludwig et al. ${ }^{4}$ showed that a special case of the sublattice problem, the random vector potential model (see Sec. II B below), has a power-law density of low (i.e., near zero) energy states

$$
\rho(E) \sim E^{-1+2 / z}
$$

parametrized by a continuously varying dynamical exponent $z$. If we define an energy-dependent length scale $L(E)$ from the density of states so that the integrated density $N(E)$ $\equiv \int_{0}^{E} d E^{\prime} \rho\left(E^{\prime}\right) \equiv L^{-2}(E)$, then Eq. (1) corresponds to dynamical scaling of the form $E \sim L^{-z}$.

In related papers, Gade and Wegner ${ }^{1,2}$ developed a fieldtheoretic description of the general sublattice problem (with random mass terms in addition to random vector potential disorder-see Sec. II B below), predicting a strongly divergent density of states

$$
\rho(E) \sim \frac{1}{E} \exp \left(-c|\ln E|^{1 / x}\right),
$$

with $x=2$, stronger than any finite- $z$ power-law Eq. (1) (these results have also been rederived very recently ${ }^{6}$ from a 
somewhat different field-theoretic analysis). This "Gade form" corresponds to a kind of activated (infinite- $z$ ) dynamical scaling

$$
|\ln E| \sim(\ln L)^{x},
$$

when $x>1$.

Finally, it is useful to note that these bipartite problems are closely related to a well-studied vortex glass problem. ${ }^{7-9}$

The field-theoretic analyses that lead to these predictions, however, do not provide a direct physical picture of the origin of the divergent DOS at the band center and the nature of the low-energy states. Developing such a picture is the main thrust of the present paper. In particular, our analysis leads us to suggest that the asymptotic scaling between the energy and the length in the general sublattice problem has the infinite- $z$ form Eqs. (2) and (3), but with a different exponent

$$
x=\frac{3}{2} \text {. }
$$

In the remainder of this section, we now outline our physical picture of the low-energy states, and summarize the basic argument that leads us to Eq. (4); for details and some relevant definitions, see Secs. II and III below.

The mechanism by which disorder produces a pileup of low-energy states is best understood by first considering the special random vector potential model. ${ }^{4}$ In the lattice version of this model, the hopping matrix elements are not independent random variables. Instead, they are given by the differences between the values of a random Gaussian field $\Phi(\mathbf{r})$ [see defining Eqs. (14) and (15)] on the two sites. This field has the statistics of a rough surface, with the variance of the difference in $\Phi$ between adjacent sites being set by a disorder strength parameter $g$. As we argue later in Sec. III, the lowenergy states at strong randomness $g \gg 1$ "live" near the extrema of the surface $\Phi(\mathbf{r})$, and the logarithms of their energies are roughly given by the corresponding relative surface heights. Such extremal properties of two-dimensional Gaussian surfaces are well characterized (see the Appendix for a summary of the relevant results); in particular, in a sample of size $L$, the corresponding prediction for the energy of the lowest state is

$$
|\ln E| \sim\left[\Phi_{\max }(L)-\Phi_{\min }(L)\right] \sim \sqrt{g} \ln L,
$$

implying power-law dynamical scaling with the dynamical exponent $z$ growing as

$$
z \sim \sqrt{g}
$$

for strong randomness $g \gg 1$. As mentioned earlier, Ref. 4 indeed predicts a power-law DOS in this model, but with the dynamical exponent

$$
z=1+\frac{g}{\pi}
$$

within the field-theoretic analysis, this result appears to be perturbatively exact to all orders in $g$. However, the above "surface argument" shows that Eq. (7) cannot hold for strong disorder, since the smallest energy in the problem cannot fall below the limits fixed by the extrema of the surface.
Indeed, we argue in Sec. IV A that there is actually a dynamical transition at $g=g_{c} \equiv 2 \pi$; at this transition, the exponent $z$ changes its behavior from the weak-disorder form (7) to the strong-disorder form (6) [see also Eq. (30)]. This dynamical transition is a counterpart of a static transition ${ }^{10,11}$ known to occur when the wave function $\exp \left(-\left[\Phi(\mathbf{r})-\Phi_{\min }\right]\right)$ (i.e., the zero-energy "pseudo-eigenstate" defined so that its peak value is 1) becomes normalizable in the limit $L \rightarrow \infty$.

Turning to the general case where the hopping matrix elements are independently random, we thus expect some power-law density of states to be produced as long as there is some "vector potential" component to the randomness, as there is. Moreover, a field-theoretic renormalization group (RG) analysis $^{6-9}$ shows that any amount of a more general "random mass" disorder (see Sec. II A below) generates additional vector potential randomness, driving the latter to stronger disorder at larger length scales and lower energy scales:

$$
g_{\text {eff }}(L) \sim \ln L
$$

The nature of the low-energy spectrum and the corresponding dynamical scaling in the general case can now be heuristically understood by considering an "effective" random vector potential model with a scale-dependent disorder strength parameter $g_{\text {eff }}(L)$ given by Eq. (8) (see Sec. IV B for a more precise argument from a different perspective using the results of Ref. 12 for a related vortex glass problem). The low-energy states in this effective problem are again associated with the extrema of some effective surface (which may be identified with the logarithm of the magnitude of the lowest-energy wave function), but the height extrema of this surface, and correspondingly the log-energies, now scale with $L$ as

$$
|\ln E| \sim z_{\text {eff }}(L) \ln L \sim \sqrt{g_{\text {eff }}(L)} \ln L \sim(\ln L)^{3 / 2},
$$

which is the proposed "modified-Gade form." [Note that the original Gade form would be obtained if the weak-disorder form for $z$, Eq. (7), were used:

$$
|\ln E| \sim z_{\text {eff }}(L) \ln L \stackrel{! ? !}{\sim} g_{\text {eff }}(L) \ln L \sim(\ln L)^{2} .
$$

However, since $g_{\text {eff }}(L)$ grows indefinitely with $L$, this form cannot be used to obtain the true asymptotic behavior.]

So far, we have only alluded to critical bipartite systems whose zero-energy states are not exponentially localized. However, it is possible to drive such a system into a different localized phase, keeping the particle-hole symmetry intact: This can be achieved, for example, by making some prescribed bonds that produce a complete dimer cover of the lattice stronger on average, thus introducing a preferred "dimerization" pattern. If this dimerization is weak, the system may remain critical, but strong dimerization will eventually drive it into a localized (insulating) state. We have found an interesting mechanism whereby disorder generates power-law contributions to the low-energy DOS in such bipartite insulating phases: The low-energy states are associated with the end points of "strings" along which the background dimer pattern is broken as shown in Fig. 1. An 


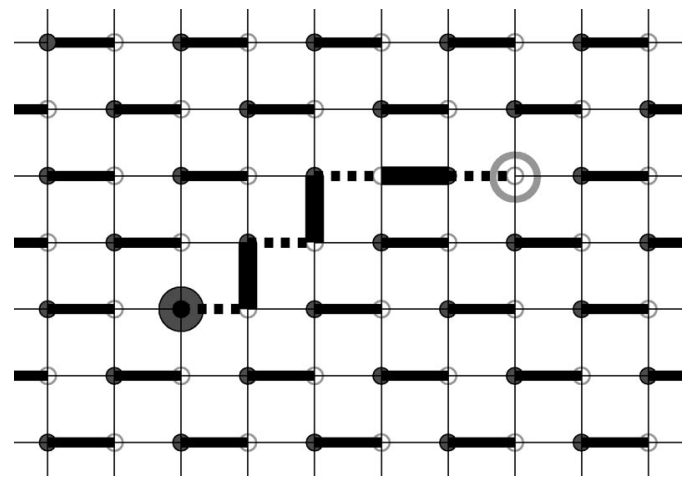

FIG. 1. "String" mechanism for generating low-energy states in the band insulator phases. Filled and open circles represent $A$ and $B$ sites, respectively. Medium thick bonds represent a particular background dimer pattern (i.e., bonds that are typically stronger), broken bonds from this set represent couplings that happened to be atypically weak in a given disorder realization, while the heavy thick bonds represent atypically strong couplings. In the above figure, there is a pair of low-energy states associated with the end points of the resulting string.

estimation of the relevant probabilities shows that this mechanism is actually operative for BPRH models in any dimension, as well as in the localized phases of the related ImRH problems (in the latter case, particular dimerization patterns appear quite naturally when describing disordered superconductors with broken spin-rotation invariance $\left.{ }^{13}\right)$. In our bipartite systems, this "Griffiths-like" mechanism can produce a power-law-divergent DOS and dominate over all other mechanisms of filling the band gap near zero energy in such localized phases.

Having introduced our principal results, we now conclude this section with an outline of the rest of the paper. Section II defines our models and reviews the connection with the vortex glass problem. Section III introduces a strongrandomness picture of the low-energy physics, motivating the more precise bounds of Sec. IV on the dynamical scaling in the system. Section V summarizes our numerical evidence in support of our analytical arguments. Finally, Sec. VI touches upon some unresolved questions and prospects for future study.

\section{MODELS, DEFINITIONS, AND THE CONNECTION WITH DIMERS}

A bipartite random hopping problem is completely specified by a single-particle Hamiltonian

$$
\hat{H}=\sum_{(\alpha \beta)}\left(t_{\alpha \beta}|\alpha\rangle\langle\beta|+\text { H.c. }\right) \equiv\left(\begin{array}{cc}
0 & \hat{t}_{A B} \\
\hat{t}_{A B}^{\dagger} & 0
\end{array}\right),
$$

where $\alpha$ and $\beta$ belong to sublattices $A$ and $B$, respectively, of some bipartite lattice. In this paper, we only consider real hopping problems in two dimensions (these are Hamiltonians of class BDI in Cartan's classification ${ }^{14}$ ), focusing attention on the following specific models: $\pi$-flux, honeycomb, and general bipartite lattice models.
Each of these models is defined as a nearest-neighbor hopping problem on an appropriate lattice, with some additional constraints on the allowed signs of the couplings: The $\pi$-flux model ${ }^{15}$ is defined on a square lattice with a requirement that there is one-half of a magnetic flux quantum per square plaquette. A convenient gauge is

$$
t_{\mathbf{j}, \mathbf{j}+\hat{\mathbf{x}}}=(-1)^{j_{y}} t_{x}(\mathbf{j}), \quad t_{\mathbf{j}, \mathbf{j}+\hat{\mathbf{y}}}=t_{y}(\mathbf{j}),
$$

where $\mathbf{j}=\left\{j_{x}, j_{y}\right\}$ labels sites of the square lattice, $\hat{\mathbf{x}}$ and $\hat{\mathbf{y}}$ are unit lattice vectors, and $t_{x}(\mathbf{j})$ and $t_{y}(\mathbf{j})$ are non-negative hopping amplitudes. The honeycomb (brickwall) lattice model ${ }^{16}$ is defined on a honeycomb lattice with non-negative hopping amplitudes. Finally, by the general lattice model, we mean a nearest-neighbor hopping model on a square or honeycomb lattice with no constraint on the allowed signs.

Naturally, the pure-system spectra in the above models can be quite different: The pure $\pi$-flux and honeycomb lattice models have Dirac points and a linearly vanishing density of states at $E=0$, while the pure rectangular lattice model has a Fermi surface with a smooth DOS near $E=0$ in the general anisotropic case and a van Hove singularity in the isotropic square lattice case. However, all these models are believed to have similar low-energy long-wavelength localization physics in the presence of randomness. In our studies, it is often convenient to work with a particular model when using a particular approach, and we switch among the models by a fair amount in what follows.

\section{A. Continuum description of models}

The pure $\pi$-flux model has two Dirac points. The corresponding weakly disordered model is described by a continuum Dirac Hamiltonian, which may be conveniently written as $^{15}$

$$
\hat{h}_{4}=\left(\begin{array}{cc}
0 & \hat{h}_{2} \\
\hat{h}_{2}^{\dagger} & 0
\end{array}\right)
$$

with

$$
\hat{h}_{2}=\sigma_{x}\left(-i v_{x} \partial_{x}+i A_{x}\right)+\sigma_{y}\left(-i v_{y} \partial_{y}+i A_{y}\right)-i V+M \sigma_{z} .
$$

In the above, the slowly varying vector and scalar potentials are implicitly defined by $2 t_{x}^{(0)}=v_{x}, 2 t_{y}^{(0)}=v_{y},-2 \delta t_{x}(\mathbf{j})$ $=A_{x}(\mathbf{j})(-1)^{j_{x}+j_{y}}+V(\mathbf{j})(-1)^{j_{x}}+\ldots, \quad-2 \delta t_{y}(\mathbf{j})=A_{y}(\mathbf{j})$ $(-1)^{j_{x}+j_{y}}+M(\mathbf{j})(-1)^{j_{y}}+\ldots$, and the lattice constant has been set to unity. A similar continuum Hamiltonian is obtained for the honeycomb lattice model.

The Dirac Hamiltonian $\hat{h}_{2}$ is non-Hermitian and contains a real random mass $M$, a random imaginary potential $-i V$, and a random imaginary gauge field. The $\{M, V\}$ terms form a "complex random mass" part, which we refer to simply as the random mass part of the disorder. In its absence, $M \equiv V$ $\equiv 0$, the full Hermitian Hamiltonian $\hat{h}_{4}$ acting on a fourcomponent field $\left\{\psi_{1}, \psi_{2}, \psi_{3}, \psi_{4}\right\}$ does not mix the pairs $\left\{\psi_{1}, \psi_{4}\right\}$ and $\left\{\psi_{2}, \psi_{3}\right\}$; the corresponding " $1-4$ " block for the $\left\{\psi_{1}, \psi_{4}\right\}$ pair has a form

$$
\hat{h}_{\mathcal{A}}=\sigma_{x}\left(-i \partial_{x}+\mathcal{A}_{1}\right)+\sigma_{y}\left(-i \partial_{y}+\mathcal{A}_{2}\right)
$$


identical to the (real) random vector potential model of Ref. 4 (here $\left\{\mathcal{A}_{1}, \mathcal{A}_{2}\right\} \equiv\left\{A_{y},-A_{x}\right\}$ ), and similarly for the "2-3" block. Here and henceforth, we will therefore refer to the $\left\{A_{x}, A_{y}\right\}$ part of $\hat{h}_{4}$ as the random vector potential part of the disorder.

\section{B. The random vector potential model on a lattice}

Consider a "random-surface" BPRH model constructed from a field $\Phi(\mathbf{r})$ and a pure system Hamiltonian with bare hopping amplitudes $\hat{t}_{A B}^{(0)}$ as follows:

$$
t_{\alpha \beta}=e^{\Phi(\alpha)} t_{\alpha \beta}^{(0)} e^{-\Phi(\beta)} .
$$

One can easily see that if $\boldsymbol{\Psi}_{A}^{(0)}$ is a zero-energy eigenstate of the pure model, then $\boldsymbol{\Psi}_{A}$ with $\Psi_{A}(\alpha)=e^{-\Phi(\alpha)} \Psi_{A}^{(0)}(\alpha)$ is a zero-energy eigenstate of the random-surface model. Moreover, it is easy to see that this construction provides a particular lattice realization of the random vector potential model, if the pure system one starts with is the nonrandom $\pi$-flux model on a square lattice. Indeed, in the continuum limit, we obtain $M \equiv V \equiv 0, \quad A_{x}=v_{x} \partial_{x} \Phi(\mathbf{r})$, and $A_{y}$ $=v_{y} \partial_{y} \Phi(\mathbf{r})$, so that $\hat{h}_{\mathcal{A}}$ of Eq. (13) becomes $\hat{h}_{\mathcal{A}}=\sigma_{x}\left(-i \partial_{x}\right.$ $\left.+\partial_{y} \Phi\right)+\sigma_{y}\left(-i \partial_{y}-\partial_{x} \Phi\right)$. Our random surface $\Phi(\mathbf{r})$ thus represents the physical (nongauge) degrees of freedom of the random vector potential, and we choose it to be Gaussian,

$$
P[\Phi] \propto \exp \left[-\frac{1}{2 g} \int d^{2} \mathbf{r}(\boldsymbol{\nabla} \Phi)^{2}\right],
$$

with dimensionless disorder strength $g$; the relevant properties of such surfaces in two dimensions are summarized in the Appendix.

A remark is in order here: In the continuum limit of the general problem, we have seen that there is a clean separation of the disorder into its random vector potential and random mass parts. However, in the original model on a lattice, such a separation is not at all obvious - the only precise statement we make at the lattice level is that a model has purely vector potential randomness when it can be put in the form given by Eq. (14). Renormalizing on a lattice in Sec. III, we will still speak of the flows of the two parts of the disorder, implicitly assuming some coarse-grained description and relying on the corresponding results for the continuum model. A more precise lattice alternative to such a hybrid RG approach is the domain wall energetics picture of Ref. 12, which we will have occasion to appeal to in Sec. IV B.

\section{Connection with random dimer problems}

There is a direct connection between the $\pi$-flux random hopping problem and a random dimer model on the same square lattice. The dimer model consists of all complete coverings (matchings) $\{\mathcal{M}\}$ of the (bipartite) lattice by the nearest-neighbor dimers; the energy of a given covering $\mathcal{M}$ is

$$
\mathcal{E}_{d}[\mathcal{M}] \equiv \sum_{\langle\alpha \beta\rangle \in \mathcal{M}} \epsilon_{\alpha \beta},
$$

where the sum is over all dimers in this covering, and $\epsilon_{\alpha \beta}$ 's are the random bond energies. The fermion-dimer connection is stated most easily for open boundary conditions: In this case, the partition function of the dimer model $Z_{d}$ $\equiv \Sigma_{\mathcal{M}} \exp \left(-\mathcal{E}_{d}[\mathcal{M}] / T_{d}\right)$ at a given dimer temperature $T_{d}$ can be written as a determinant of an appropriate connection matrix $\hat{t}_{A B}, Z_{d}[A, B]=\operatorname{det} \hat{t}_{A B}$, where

$$
t_{\alpha \beta}= \pm e^{-\epsilon_{\alpha \beta} / T_{d}}
$$

and the signs of the hopping amplitudes are chosen exactly as in the $\pi$-flux model. ${ }^{17,18}$ The honeycomb lattice model with non-negative bonds has a similar dimer connection (our definitions of the $\pi$-flux and honeycomb models were made precisely with this in mind), but the more general lattice model has no such direct connection.

\section{Localized bipartite hopping systems}

The commonly studied bipartite hopping models (e.g., all of the above models with some generic distributions of random hopping amplitudes) are found to be critical, by which we mean that the behavior at $E=0$ is neither truly delocalized nor truly (exponentially) localized. Abusing language somewhat, we will often refer to a system in such a critical state as being in a "delocalized" or "metallic" phase-this serves to emphasize the distinction between such a phase and truly localized insulating phases that are obtained, e.g., by introducing strong dimerization in the couplings. The puresystem counterparts of these localized phases are typically gapped band insulators. In our studies, we will specifically consider only one such localized phase produced by introducing "staggered" dimerization on the square lattice (see Fig. 1); the honeycomb lattice version of this is shown in Fig. 5(a).

\section{ON THE ORIGIN OF LOW-ENERGY STATES}

We begin by noting that the Gade-like dynamical scaling Eq. (3) with $x>1$ suggests that the effective value of disorder scales to infinity at asymptotically low energies (dynamical exponent $z=\infty$ ), albeit very weakly. This motivates us to try a strong-randomness RG description. ${ }^{19,13}$

\section{A. Strong-randomness RG}

Consider the bipartite hopping Hamiltonian (11). The eigenstates of $\hat{H}$ occur in pairs with energies $\pm E$, and the strong-randomness RG proceeds by eliminating, at each step, such a pair of states with energies near the top and the bottom of the band: One finds the largest (in absolute value) coupling in the system, say $t_{1,2}$ connecting sites $1 \in A$ and $2 \in B$; this defines the bandwidth $2 \Omega=2 \max \left\{\left|t_{\alpha, \beta}\right|\right\}$. If the distribution of the couplings is broad, the two eigenstates of the two-site problem $\hat{H}[1,2]$ with energies $\pm \Omega$ will be good approximations to eigenstates of the full $\hat{H}$, since the couplings $t_{1, \beta}$ and $t_{2, \alpha}$ of the pair to the rest of the system will 
typically be much smaller. These couplings can then be treated perturbatively, and eliminating the two high-energy states living on the sites 1 and 2 gives us the following effective couplings between the remaining sites:

$$
t_{\alpha, \beta}^{\prime}=t_{\alpha, \beta}-t_{\alpha, 2} t_{1,2}^{-1} t_{1, \beta}
$$

The renormalized Hamiltonian is again a bipartite hopping problem, but with two fewer sites; in particular, no diagonal terms are generated. This procedure is then iterated to reach lower and lower energy.

As a first attempt, we implemented this RG numerically in both localized and metallic phases. In the localized phases, the RG indeed provides a qualitatively and quantitatively accurate description of the low-energy physics, with the relative "decimation errors" diminishing quickly at low energies; in this case, the RG is thus a consistent scheme that correctly describes the Griffiths effects, which are analyzed in detail below. However, in the delocalized phase, the observed flow to stronger disorder is very weak, and the consistency of the RG is less clear. We do not address this question of self-consistency in detail here (that is, in which regimes - if at all-the system flows to stronger disorder, with the RG becoming more and more accurate). Rather, in the metallic phase, we view the RG as providing a heuristic picture that complements the more precise arguments we present later. Note, however, that the RG actually contains some exact and useful information for any strength of the disorder: ${ }^{13}$ The rule Eq. (18) is an exact transformation for the zero-energy wave function, and as such provides useful information on the $E=0$ localization properties. Also, running this RG corresponds to employing the Sturm sequence method for calculating the integrated density of states at $E$ $=0$ using a particular order that is, without any a priori knowledge, numerically most reliable; the intermediate terms that appear in this process at a particular length scale give us a rough idea about the corresponding energy scale in the problem.

\section{B. On the origin of states}

The strong-randomness RG associates the low-energy states of the original Hamiltonian with the sites that "survive" down to the corresponding energy. Such a "free" site- say, an $A$ site-is found if all the $B$ sites in its immediate neighborhood happen to be "locked" (by stronger bonds) into pairs with some other $A$ sites. Then, this free $A$ site can only couple to the next available $B$ sites, which are far away, and the corresponding effective couplings are relatively weak since they are mediated by a substantial intervening pair-locked region.

At first it is difficult to see how such free sites and the corresponding low-energy states can be produced often enough in dimensions $d>1$ : Naively, the energy scale associated with a pair $\{\alpha, \beta\}$ of "isolated" sites distance $l$ apart is $\sim e^{-c_{E} l}$. Now, it may seem that in order to produce such a pair we need of order $l^{d}$ specific events-for example, we can imagine having a particular pairing pattern for all the other sites in the neighborhood of the two unpaired sites $\alpha$ and $\beta$-and this has a very low probability $P \sim e^{-c_{p} l^{d}}$ in $d$ $>1$.

In the dimerized band insulator phase, however, there is an insulating background to start with, and to produce such a pair, one only needs to "break" the background dimer pattern along a string joining the two sites, i.e., of order $l$ specific events (see Fig. 1), with the resulting occurrence rate $\sim e^{-c_{p}^{\prime} l}$ high enough to give a power-law contribution to the low-energy density of states. Our numerical RG and exact diagonalization studies confirm that this is indeed what happens in such band insulator phases. Furthermore, this "Griffiths-string" argument goes through for localized phases in any dimension.

In the "delocalized" phase, on the other hand, we do not have such an insulating background. However, the local properties of the "isolating" region need not be very "sharp," as was assumed in making the estimate for the probability of occurrence $\sim e^{-c_{p} l^{d}}$. Rather, there might be some smooth "textures" of the random pairing (dimer) pattern producing such isolated sites (defects), and these may occur with much higher probability.

To be more specific, first consider the "random-surface" model Eq. (14) (i.e., the random vector potential model) in the limit of strong disorder $g \gg 1$. The RG, being exact for the zero-energy wave function, preserves the random-surface character of the problem leaving $\Phi(\mathbf{r})$ unchanged: the randomness in the effective couplings that are generated by the RG rule Eq. (18) is of the same random-surface form Eq. (14) with the same $\Phi(\mathbf{r})$. Thus, at each stage of the RG, the magnitude of the coupling between any two remaining sites $\alpha$ and $\beta$ is roughly $\sim e^{\Phi(\alpha)-\Phi(\beta)}$. Since the RG eliminates the stronger couplings first, an $A$-site $\alpha_{\min }$ that is a local minimum of $\Phi(\mathbf{r})$ cannot be decimated out until the "probing" log-energy scale is large enough to "see" the next even lower minima: Indeed, before this happens, the other $A$ sites in the neighborhood of $\alpha_{\min }$, having higher $\Phi$, are more strongly coupled to any available $B$ sites in the same neighborhood. The same holds for any $B$-site $\beta_{\max }$ that is a local maximum of $\Phi(\mathbf{r})$. As a result, within our strongrandomness RG picture, the low-energy states of the problem are associated with the local extrema of the surface $\Phi(\mathbf{r})$, while their effective log-energies are given by the corresponding surface height differences. In particular, in a finite system, the smallest energy scale is associated with the global extrema of $\Phi(\mathbf{r})$. The arguments leading to Eqs. (5) and (6) of the Introduction follow now.

Thus, within our somewhat heuristic RG approach, "isolated" sites are produced often enough to result in a powerlaw contribution to the density of states, with the dynamical exponent $z \sim \sqrt{g}$ in the strong-randomness regime. Here, we are ignoring effect of running the RG transformation on the pure model itself-this will change the actual value of the exponent $z$, but not the $\sim \sqrt{g}$ disorder contribution for $g$ $\gg 1$. For more precise bounds that complement these heuristics and lead to identical conclusions, see Sec. IV A.

In the general case, the heuristic RG arguments Eqs. (8) and (9) in the Introduction now follow and yield the modi- 
fied Gade form Eqs. (2) and (3) with $x=\frac{3}{2}$; more precise arguments leading to identical conclusions are presented in Sec. IV B. However, it should be emphasized again that we have not found a clear-cut separation of the random vector potential and random mass parts of the disorder at the lattice level. We, therefore, have to rely on the results of a different field-theoretic RG analysis ${ }^{7,9}$ when discussing how the two types of randomness flow and affect each other (note that a similar somewhat heuristic argument was used in Ref. 20 to obtain crossover scales in the vortex glass problem).

We conclude this discussion of the origin of low-energy states with some miscellaneous observations: Recall that the surface in the random vector potential case is also the logarithm of the pseudo-zero-energy-wave function $\Psi_{A}(\alpha)$ $\sim e^{-\Phi(\alpha)}$. Given that the RG rule is an exact transformation for such a wave function in the general case as well, the above analysis suggests that the $E \neq 0$ low-energy states in the general problem are again related to the extremal properties of this $E=0$ wave function. It is also useful to note the predictions of this "surface" mechanism in other dimensions. In one dimension, any disorder is of purely "longitudinal" (random-surface) type, and the Gaussian surface is more rough, with

$$
|\ln E| \sim \Phi_{\max }(L)-\Phi_{\min }(L) \sim \sqrt{L} ;
$$

this is precisely the Dyson critical scaling for the 1D chain. For bipartite systems that are finite in all but one direction (such as ladders ${ }^{21,22}$ ), the DOS again has the Dyson form at special delocalized critical points in the phase diagram, since the corresponding surface "looks," at long wavelengths, as a 1D Gaussian surface. On the other hand, in dimensions three and higher the Gaussian surface is basically flat, and this mechanism cannot generate power-law contributions to the density of states. Much of what has been said above for the two-dimensional problem is therefore very specific to $2 \mathrm{D}$.

\section{From low-energy states to optimized defects}

We now establish a suggestive connection between the low-energy states in the bipartite hopping problem and monomers (defects) in the corresponding random dimer problem. The dimer connection leads us to a heuristic "dimer RG" prescription for the low-energy states-this dimer RG approach provides us with a very useful physical picture that underlies the more precise analysis of Sec. IV.

Consider running the above "zero-energy" RG in some specified order. (The naive strong-randomness RG described earlier uniquely prescribes a particular order by picking the strongest effective bond at each step, but it is important to realize that in the absence of any rapid flows to stronger effective disorder, there is nothing "sacred" about this ordering, and other sensible choices are possible.) We denote the $A$ and $B$ sites decimated out at a given stage of the RG as $\mathfrak{A}=\left\{a_{1}, \ldots, a_{n}\right\} \subset A$ and $\mathfrak{B}=\left\{b_{1}, \ldots, b_{n}\right\} \subset B$, respectively. The effective coupling between any two remaining sites $\alpha$ and $\beta$ is given by

$$
t_{\alpha, \beta}^{\prime}=t_{\alpha, \beta}-\hat{t}_{\alpha, \mathfrak{B}} \hat{t}_{\mathfrak{A}, \mathfrak{B}}^{-1} \hat{t}_{\mathfrak{A}, \beta} .
$$

Here, $\hat{t}_{\mathfrak{C}, \mathfrak{D}}$ is a matrix of the original bonds joining two subsets $\mathfrak{C} \subset A$ and $\mathfrak{D C} B$ (thus, $\hat{t}_{a, \mathfrak{B}}$ is a row vector, $\hat{t}_{\mathfrak{A}, \beta}$ is a column vector, and $\hat{t}_{\mathfrak{A}, \mathfrak{B}}$ is an $n \times n$ matrix). Expression (20) follows readily if we note how the RG "solves" for the zeroenergy wave function by eliminating any reference to the decimated sites, and this holds for any order of the decimations. Furthermore, it is easy to check that this may be rewritten as

$$
t_{\alpha, \beta}^{\prime}=\frac{\operatorname{det} \hat{t}_{\alpha+\mathfrak{A}, \beta+\mathfrak{B}}}{\operatorname{det} \hat{t}_{\mathfrak{A}, \mathfrak{B}}},
$$

where $\alpha+\mathfrak{A} \equiv\left\{\alpha, a_{1}, \ldots, a_{n}\right\}$, and similarly for $\beta+\mathfrak{B}$. It is this form that we find useful below.

Now, recall from Sec. IIC that for any two such sets $\mathfrak{C}$ and $\mathfrak{D}$ of equal cardinality

$$
\operatorname{det} \hat{t}_{\mathfrak{C}, \mathcal{D}}=\sum_{\mathcal{M}} \operatorname{sgn}(\mathcal{M}) \prod_{(\alpha \beta) \in \mathcal{M}} t_{\alpha \beta},
$$

where the sum is over all complete dimer coverings of $\mathfrak{C} \cup \mathfrak{D}$, and $\operatorname{sgn}(\mathcal{M})$ denotes the appropriate permutation sign for matching $\mathcal{M}$. Each term of the sum has a form $\pm \exp$ $\left(-\mathcal{E}_{d}[\mathcal{M}] / T_{d}\right)$, with the dimer bond energies $\epsilon_{\alpha \beta}$ defined from $\left|t_{\alpha \beta}\right|=\Omega_{0} e^{-\epsilon_{\alpha \beta} / T_{d}}$. In the limit of infinite randomness, or equivalently, zero temperature of the dimer system $T_{d}$ $=0$, one term - the ground state dimer covering — dominates the whole sum; obviously, the permutation signs play no role in this limit.

Consider first this infinite-randomness limit. The largest term in $\operatorname{det} \hat{t}_{\alpha+\mathfrak{A}, \beta+\mathfrak{B}}$ corresponds to the optimal (groundstate) dimer covering of $\{\alpha+\mathfrak{A}\} \cup\{\beta+\mathfrak{B}\}$, while the largest term in $\operatorname{det} \hat{t}_{\mathfrak{A}, \mathfrak{B}}$ corresponds to the optimal covering of $\mathfrak{A} \cup \mathfrak{B}$. The two optimal coverings "differ" by a "string" connecting the "added" sites $\alpha$ and $\beta$, while the effective coupling $t_{\alpha, \beta}^{\prime}$ in this infinite-randomness limit, Eq. (21), is determined precisely by "propagating" along the string $t_{\alpha, \beta}^{\prime}=t_{1} t_{3} \cdots t_{2 k+1} /\left(t_{2} \cdots t_{2 k}\right)$. Note that this string is the optimal (i.e., corresponding to the largest possible $\left|t^{\prime}\right|$ ) such path from $\alpha$ to $\beta$ utilizing the sites decimated out.

This suggests the following infinite-randomness "dimer RG" sequence: At the $n$th stage of such a dimer RG analysis, we find the ground-state configuration $\mathcal{M}_{n}^{(\mathrm{gs})}$ with $n$ dimers, defining $\mathcal{E}_{n}^{(\mathrm{gs})} \equiv \mathcal{E}_{d}\left[\mathcal{M}_{n}^{(\mathrm{gs})}\right]$. The $2 n$ sites covered by dimers at this stage are to be thought of as having already been decimated. At each step of this RG, precisely two sites are "integrated out" since the optimal $(n+1)$-dimer covering differs from the optimal $n$-dimer covering by two added sites (and the connecting string). We now need to provide, for the random hopping problem, some notion of the bandwidth (i.e. the value of the cutoff energy) corresponding to each stage. A natural choice is to associate the effective hopping amplitude $\left|t^{\prime}\right|=\Omega_{0} \exp \left[-\left(\mathcal{E}_{n+1}^{(\mathrm{gs})}-\mathcal{E}_{n}^{(\mathrm{gs})}\right) / T_{d}\right]$ with the two sites "eliminated" in going from stage $n$ to $n+1$-this then specifies the value of the cutoff in the hopping problem at this RG step. The monotonicity property $\mathcal{E}_{n+1}^{(\mathrm{gs})}-\mathcal{E}_{n}^{(\mathrm{gs})} \geqslant \mathcal{E}_{n}^{(\mathrm{gs})}-\mathcal{E}_{n-1}^{(\mathrm{gs})}$ guarantees that the cutoff indeed decreases monotonically as the 
RG proceeds. Note, however, that the "pairings" of the sites decimated out are somewhat subtle; these do not stay rigid as the RG proceeds, and can change each time we decimate more sites. Furthermore, there is no simple relationship in general between the sequence of decimations within this dimer RG and that in the naive strong-disorder RG sequence described earlier.

Of course, this can all be reformulated starting from the ground-state complete dimer covering (with $N=L^{2} / 2$ dimers) of the whole lattice, and then adding pairs of monomers (defect pairs) into the system in the most optimal way. In particular, within this heuristic picture, the lowest-energy state in the hopping problem corresponds precisely to the first such pair, with the corresponding estimate

$$
\left|t^{\prime}\right|_{\min }^{(\text {dimer RG) }} \equiv \Omega_{0} \exp \left(\frac{\mathfrak{e}_{\mathrm{opt} \text { def }}}{T_{d}}\right) ;
$$

here $\mathfrak{e}_{\mathrm{opt} \text { def }} \equiv \mathcal{E}_{N-1}^{(\mathrm{gg})}-\mathcal{E}_{N}^{(\mathrm{gs})}$ is the optimized (lowest energy) defect pair energy, and all of the foregoing assumes the system has complete coverings to start with. ${ }^{23}$

Returning to the finite-randomness case, there are several issues that stand out when trying to relate the low-energy states of the hopping problem to the monomers in the corresponding finite-temperature random dimer system. The determinant $\operatorname{det} \hat{t}_{\mathfrak{A}, \mathfrak{B}}$ and the dimer partition function $Z_{d}[\mathfrak{A}, \mathfrak{B}]$ still contain the same (in absolute value) terms, but now the dimer ground state by itself does not determine the corresponding sums, so the permutation signs become important. Even if we use the dimer partition function only to bound the absolute value of the determinant, we are still faced with the dimer problem at finite temperature, which now has significant entropic contributions, since local moves from the ground state cost only finite energy. In this situation, we can no longer think solely in terms of the ground-state pairing when attempting to estimate $Z_{d}[\mathfrak{A}, \mathfrak{B}]$.

However, we are primarily interested in the ratios of determinants, such as $\operatorname{det} \hat{t}_{A, B}$ and $\operatorname{det} \hat{t}_{A-\alpha, B-\beta}$, i.e., the difference in the corresponding free energies of the dimer system without defects and with two defects. Since a large part of the entropic terms in the two systems comes from the same local moves, the ground-state energy difference of the two may still be a relevant object. Put another way, we need to consider the effective thermodynamics of the defects themselves, i.e., with all free energies measured relative to the defect-free system. As we shall see within a more precise formulation in Sec. IV B, this effective thermodynamics is indeed ground-state dominated, allowing us to make fairly precise statements for finite values of bare disorder as well.

\section{IV. "VARIATIONAL" BOUNDS ON THE STATES NEAR THE BAND CENTER}

Motivated by these heuristic RG considerations, we now establish more rigorous bounds on the dynamical scaling in the system. Consider the bipartite Hamiltonian (11) in a finite sample and assume that there are no zero-energy states and no edge states; a concrete realization would be some regular lattice block with some nice boundary conditions, e.g., the $\pi$-flux model on an $L \times L$ square with free boundary conditions (bc) and even $L$. The smallest positive energy $E_{\text {min }}$ $\equiv E_{\min }^{+}$of $\hat{H}$ is then given as

$$
E_{\min }=\frac{1}{\left\|\hat{t}_{A B}^{-1}\right\|} .
$$

Here, $\|\hat{C}\|$ is the matrix norm of an operator $\hat{C}$, and satisfies the inequalities

$$
\max _{i, j}\left|C_{i j}\right| \leqslant\|\hat{C}\| \leqslant \sqrt{\sum_{i, j}\left|C_{i j}\right|^{2}} \leqslant \sum_{i, j}\left|C_{i j}\right| .
$$

Expressions (24) and (25) formalize our earlier, more heuristic association of low-energy states with optimized defects in the dimer problem. Indeed, we have

$$
\left(\hat{t}_{A B}^{-1}\right)_{\beta \alpha}= \pm \frac{\operatorname{det} \hat{t}_{A-\alpha, B-\beta}}{\operatorname{det} \hat{t}_{A B}},
$$

which is precisely the previously encountered ratio of the determinants, and the above inequalities suggest that the two "defect" sites $\alpha$ and $\beta$ should be chosen in some optimal way.

Below, we use these inequalities to obtain, most importantly, lower bounds for the smallest positive energy. ${ }^{24}$

\section{A. The random vector potential model}

Applying the above bounds to the "random-surface" model Eq. (14), we obtain, in particular,

$$
\left\|\hat{t}_{A B}^{-1}\right\|^{2} \leqslant \sum_{\alpha, \beta} \exp [2 \Phi(\beta)-2 \Phi(\alpha)]\left|G_{0}(\beta, \alpha)\right|^{2},
$$

where $G_{0}(\beta, \alpha) \equiv\left(\hat{t}_{0}^{-1}\right)_{\beta \alpha} \equiv\left\langle\beta\left|\hat{H}_{0}^{-1}\right| \alpha\right\rangle$ is the $E=0$ Green's function (propagator) for the pure lattice problem. The propagator $G_{0}$ can be calculated explicitly for each particular pure system. In the following, we consider specifically the $\pi$-flux lattice model with free bc; the lattice propagator (Dirac fermions) can be found, e.g., in Ref. 25, but here we need only note that $G_{0}\left(\mathbf{r}_{1}, \mathbf{r}_{2}\right)$ is bounded and behaves as $\left|G_{0}\left(\mathbf{r}_{1}, \mathbf{r}_{2}\right)\right| \sim\left|\mathbf{r}_{1}-\mathbf{r}_{2}\right|^{-1}$ for large $\left|\mathbf{r}_{1}-\mathbf{r}_{2}\right| \gg 1$. A simple (completely rigorous) bound $\left\|\hat{t}_{A B}^{-1}\right\| \leqslant$ const $\times \mathfrak{Z}(L)$ immediately follows from the boundedness of $G_{0}$. Here, $\mathfrak{Z}(L)$ is the partition function for a classical particle living in a reduced potential $V(\mathbf{r}) /\left(k_{B} T\right)=2 \Phi(\mathbf{r})$ - see the Appendix, where we also summarize other relevant properties of such 2D Gaussian surfaces. Furthermore, it is actually possible to provide stronger bounds-below we discuss this separately for the strong-randomness $\left(g>g_{c} \equiv 2 \pi\right.$ ) and weak-randomness ( $g$ $<g_{c}$ ) regimes (see the Appendix for the significance of $g_{c}$ and the distinction between the two regimes in the randomsurface problem).

In the strong-randomness regime, we expect that the sum (27) is dominated by the $\beta$ 's near the maxima of $\Phi(\mathbf{r})$ and the $\alpha$ 's near the minima of $\Phi(\mathbf{r})$ [analogous to Eq. (A3) for $\mathfrak{Z}(L)]$. We also expect that these extrema are separated by 
distances of order $L$ (the precise statement would be that the distance between the global maximum and minimum of $\Phi(\mathbf{r})$ is $L$ times a random number of order one). This suggests that

$$
\left\|\hat{t}_{A B}^{-1}\right\| \leqslant(\text { num factor }) \times \frac{e^{\Phi_{\max }(L)-\Phi_{\min }(L)}}{L} \sim \frac{\mathfrak{Z}(L)}{L} .
$$

The expected uncertainty in the right-hand side (rhs) of this expression from one disorder realization to another is simply a random $O(1)$ numerical factor (num factor). [Note that even though the above estimate of the sum (27) does not constitute a rigorous proof, it is fairly robust-moreover, a more formal proof is likely possible along the lines of Refs. 10 and 11.] Since the sum in the estimate is essentially dominated by few terms, this also provides a lower bound for $\left\|\hat{t}_{A B}^{-1}\right\|$, that differs from the upper bound (28) only by an $O$ (1) numerical factor

$$
\left\|\hat{t}_{A B}^{-1}\right\| \geqslant \max _{\alpha, \beta}\left|\left(\hat{t}_{A B}^{-1}\right)_{\beta \alpha}\right|=\operatorname{const} \times \frac{e^{\Phi_{\max }(L)-\Phi_{\min }(L)}}{L} ;
$$

the bound is rigorous (and completely general) since the distance between the global extrema cannot exceed the sample size. Thus, the smallest positive energy is expected to scale with the system size as

$$
E_{\min }=(\text { num factor }) \times \frac{L}{\mathfrak{Z}(L)} \sim \frac{(\ln L)^{(3 / 2) \sqrt{\left(g / g_{c}\right)}}}{L^{4 \sqrt{\left(g / g_{c}\right)}-1}} .
$$

In particular, for the dynamical exponent we obtain

$$
z=4 \sqrt{\left(g / g_{c}\right)}-1, \quad g>g_{c} .
$$

This is our central result; pictorially, the surface $\Phi(\mathbf{r})$ simply does not allow the low-energy states to fall beyond its extrema. [In Eq. (29) we have also included the logarithmic correction from Ref. 11 to show that the minimal energy is somewhat larger than expected from the simple- $z$ power law; note, however, that the actual form of such log corrections can, in general, depend on the boundary conditions used. ${ }^{11}$ ]

In the weak-randomness regime, the bound Eq. (27) is not as precise. For instance, in the pure-system $(g=0)$ case, we obtain

$$
\sum_{\alpha, \beta}\left|G_{0}(\beta, \alpha)\right|^{2}=\operatorname{Tr} \hat{H}_{0}^{-2} \sim L^{2} \ln L,
$$

i.e., $E_{\min } \geqslant L^{-1}(\ln L)^{-1 / 2}$, which gives the correct exponent $z$ $=1$, but is certainly an underestimate. Similarly, in the $g$ $\neq 0$ case, we can prove that for any $\eta>0$

$$
\left\|\hat{t}_{A B}^{-1}\right\| \leqslant \text { const } \times L^{1+2 g / g_{c}+\eta}
$$

with probability one in the limit $L \rightarrow \infty$. The proof (following the lines of Ref. 10) applies the inequality $P(X \geqslant K)$ $\leqslant\langle X\rangle / K$, valid for any non-negative random variable $X$, to the particular $X$ equal to the rhs of Eq. (27). Thus, the dynamical exponent $z$ obeys the following bound quite generally:

$$
z \leqslant 1+\frac{2 g}{g_{c}} .
$$

Note that this result is valid in the strong disorder regime as well-however, in this case an individual realization essentially never samples the tails of the distributions that determined the average $\langle X\rangle$, and our earlier treatment leading to Eq. (30) is more appropriate and provides a much sharper bound.

For weak randomness $g<g_{c}$, we can also give a plausible argument for the opposite inequality $z \geqslant 1+2 g / g_{c}$. It suffices to provide an upper bound for $E_{\min }$, and a good trial wave function for this seems to be

$$
\overrightarrow{\boldsymbol{\psi}}_{B}=\hat{t}_{A B}^{-1} e^{-\Phi(\overrightarrow{\mathbf{A}})},
$$

with $E_{\min } \leqslant\left\|\hat{t}_{A B} \overrightarrow{\boldsymbol{\psi}}_{B}\right\| /\left\|\overrightarrow{\boldsymbol{\psi}}_{B}\right\|$ bounded by

$$
E_{\min }^{2} \leqslant \frac{\sum_{\alpha} e^{-2 \Phi(\alpha)}}{\sum_{\beta} e^{2 \Phi(\beta)}\left(\sum_{\alpha} G_{0}(\beta, \alpha) e^{-2 \Phi(\alpha)}\right)^{2}} .
$$

To translate this into an actual lower bound on $z$, we first estimate the sum over $\alpha$ sites in the denominator as

$$
\left|\sum_{\alpha} G_{0}(\beta, \alpha) e^{-2 \Phi(\alpha)}\right| \sim \sum_{\alpha}\left|G_{0}(\beta, \alpha)\right| e^{-2 \Phi(\alpha)} .
$$

This estimate is clearly reasonable, since $G_{0}\left(\mathbf{r}_{1}, \mathbf{r}_{2}\right)$ is some fixed function, while the sums involving $e^{-2 \Phi(\alpha)}$ over macro-subregions (with $G_{0}>0$ and $G_{0}<0$ ) always have $O$ (1) random numerical factors [see, e.g., expressions for $\mathfrak{Z}(L)$ in the Appendix]. Now, using $\left|G_{0}\right| \geqslant L^{-1}$ we obtain $E_{\min } \leqslant$ (num fact) $\times L / \mathcal{Z}(L)$, which gives the desired coinciding lower bound for $z$ in the weak-disorder regime (note that this also provides a similar coinciding lower bound in the strong-disorder regime, and thus reproduces our result for $z$ in this case).

We conclude with two comments on the results obtained here. First, although we cannot give a proof, we expect that the expression for $z$ in the weak-disorder regime

$$
z=1+\frac{2 g}{g_{c}}, \quad g<g_{c},
$$

is exact without any logarithmic corrections. Our expectation is bolstered by the fact that Eq. (37) coincides with the original result of the weak-disorder replica analysis of Ref. 4 (which is known to give exact results also for the static quantities of the Appendix in the regime $g<g_{c}$ ). Moreover, the two expressions for $z(g)$, Eqs. (30) and (37), join continuously and smoothly at $g_{c}$ [and we expect that $E_{\min }$ $=($ num factor $) \times L / \mathcal{Z}(L)$ is valid for any disorder strength].

Second, note that the above results are, strictly speaking, valid only for the exponent $z_{1}$ that determines the scaling of the smallest gap in the system. However, this is not expected to be a serious issue: Thus, having the upper bounds on $E_{\min }(L)$, we are guaranteed a bulk density of states with the DOS dynamical exponent $z_{\text {DOS }}$ at least as large. Moreover, we expect quite generally that there is only one dynamical exponent in the system $z_{\mathrm{DOS}} \equiv z_{1} \equiv z$. Some numerical checks 


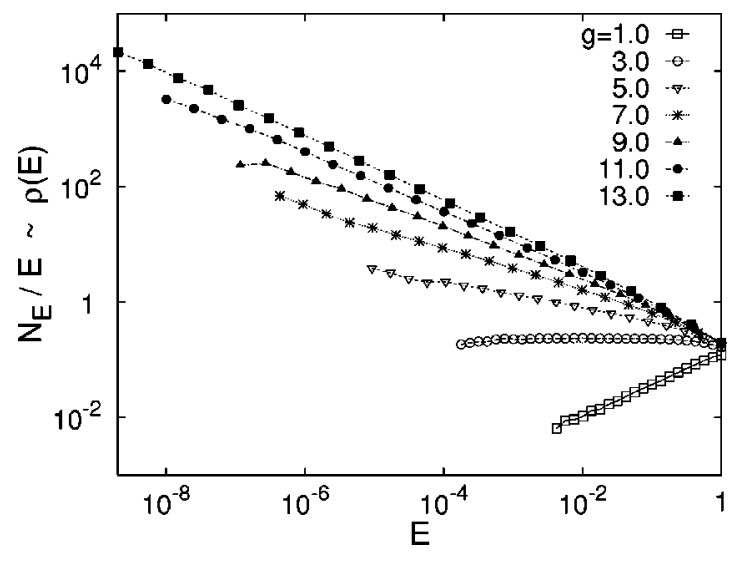

FIG. 2. Density of states in the $\pi$-flux model with the "randomsurface" disorder [see Eq. (14)] calculated for $192 \times 192$ system with open boundary conditions, for varying disorder strength $g$. The data is averaged over ten disorder realizations. Note that the plots have some curvature for strong disorder-this is probably due to the logarithmic corrections to the power laws in this regime.

for the bulk density of states are shown in Figs. 2 and 3, and these are in good agreement with the above expectations. (See also Ref. 26 studying essentially identical dynamical freezing phenomenon for random walks in random logcorrelated potentials in one and two dimensions.)

\section{B. Scaling of the lowest energy in the general case}

In general, we can always bound the numerator in Eq. (26) by the corresponding dimer partition function with two fixed monomers $\alpha$ and $\beta$ as $\left|\operatorname{det} \hat{t}_{A-\alpha, B-\beta}\right| \leqslant Z_{d}[A-\alpha, B-\beta]$. We now specialize to the $\pi$-flux model with free bc (or any other model with a direct connection to dimers), so that for the lattice with no defects we have $\left|\operatorname{det} \hat{t}_{A B}\right|=Z_{d}[A, B] \equiv Z_{N}$, with $N=L^{2} / 2$ the total number of dimers. In this case, we do not have to worry about the denominator at all, obtaining, e.g.,

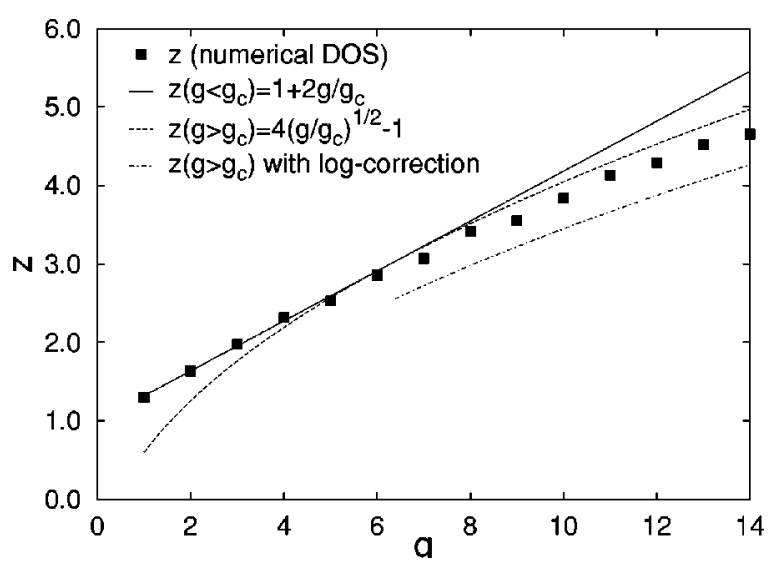

FIG. 3. Dynamical exponent for the $\pi$-flux model with the random-surface disorder extracted from the data of Fig. 2. The additional lines show theoretical predictions in the two regimes (with $\left.g_{c}=2 \pi\right)$, and also a rough estimate $z_{\mathrm{eff}}(L)=z(\infty)$ $-(3 / 2) \sqrt{g / g_{c}}(\ln \ln L) /(\ln L)$ of the $\log$ correction in the strongdisorder regime for the specific system size studied.

$$
\left\|\hat{t}_{A B}^{-1}\right\| \leqslant \frac{\Sigma_{\alpha \beta} Z_{d}[A-\alpha, B-\beta]}{Z_{d}[A, B]}=\frac{Z_{N-1}}{Z_{N}}
$$

where $Z_{N-1}$ is the partition function of the dimer problem with two free defects allowed to be anywhere on the lattice.

Now, this ratio of partition functions describes the effective finite-temperature thermodynamics of a pair of defects (i.e., with all contributions to the free energy measured relative to that of the dimer system with no defects). The analysis of Ref. 12 suggests that this effective finite-temperature thermodynamics is equivalent to the thermodynamics of a pair of classical charged particles (with opposite charges for $A$ and $B$ monomers) in a random "electrostatic" potential $v(\mathbf{r})$ with average correlations

$$
\overline{\left[v(\mathbf{r})-v\left(\mathbf{r}^{\prime}\right)\right]^{2}} \sim \ln ^{2}\left|\mathbf{r}-\mathbf{r}^{\prime}\right| .
$$

The extremal properties of this random surface $v(\mathbf{r})$ have also been characterized in Ref. 12. In a finite box of linear size $L$, we have for the average and the standard deviation of the distribution of the global extrema

$$
\overline{v_{\max }} \sim(\ln L)^{3 / 2}, \quad \sigma\left(v_{\max }\right) \sim(\ln L)^{1 / 2} .
$$

Reasoning as in Ref. 11, we see that these extremal properties immediately imply

$$
\frac{Z_{N-1}}{Z_{N}} \leqslant L^{4} \exp \left[\frac{v_{\max }-v_{\min }}{T_{d}}\right] \leqslant \exp \left[\operatorname{const} \times(\ln L)^{3 / 2}\right],
$$

and correspondingly for the lower bound on the smallest positive energy

$$
\ln E_{\min } \geqslant- \text { const } \times(\ln L)^{3 / 2} \text {. }
$$

In the above, making the positive number "const" slightly larger takes care of any prefactors $L^{r}$ and any uncertainty $o\left((\ln L)^{3 / 2}\right)$ in $v_{\max }$. This result is a simple manifestation of ground-state dominance ${ }^{11}$ for the finite-temperature partition sum of particles moving in the random potential $v(\mathbf{r})$ with correlations Eq. (39). The above crude estimates are good enough to reach the main conclusion, Eq. (42), precisely due to this, even though the ground-state dominance is a very weak one. Since a few defect positions effectively determine the above estimates, we expect that Eq. (42) provides not only the lower bound but also the actual leading term in the logarithm of the smallest positive energy in the system. This immediately leads to the modified Gade scaling proposed in the Introduction - our analysis here thus provides strong evidence in support of the RG arguments outlined in the Introduction.

To get some idea about the subleading terms and, in particular, the relationship between the actual lowest gap, the intermediate bound Eq. (38) obtained from the effective thermodynamics of a free defect pair, and the heuristic dimer RG "estimate" Eq. (23) from the optimized defect pair, it is instructive to consider, from this perspective, the random vector potential model in the strong-randomness regime $g \gg 1$. 
Recall first our earlier direct estimate of $\left\|\hat{t}_{A B}^{-1}\right\|$, i.e., the lhs of Eq. (38). Using the Dirac fermion propagator for $\hat{t}_{0}^{-1}$, we have

$$
\left|\left(\hat{t}_{A B}^{-1}\right)_{\beta \alpha}\right| \approx \frac{\text { const }}{\left|\mathbf{r}_{\beta}-\mathbf{r}_{\alpha}\right|} \times e^{\Phi(\beta)-\Phi(\alpha)} .
$$

Invoking the ground-state dominance in the strongrandomness regime, we obtain $\left\|\hat{t}_{A B}^{-1}\right\| \sim L^{-1} e^{\Phi_{\max }-\Phi_{\min }}$.

Consider now the relative partition sum $Z_{N-1} / Z_{N}$ for a free defect pair, i.e., the rhs of Eq. (38). In this "randomsurface" case, for a given fixed configuration of monomers, any dimer covering has the same energy, and the partition function is essentially purely configurational (entropic). Thus, for two fixed defects $\alpha$ and $\beta$, we have

$$
\frac{Z_{d}[A-\alpha, B-\beta]}{Z_{d}[A, B]} \approx \frac{\text { const }}{\left|\mathbf{r}_{\beta}-\mathbf{r}_{\alpha}\right|^{1 / 2}} \times e^{\Phi(\beta)-\Phi(\alpha)},
$$

since the relative number of dimer configurations decays as $\left|\mathbf{r}_{\beta}-\mathbf{r}_{\alpha}\right|^{-1 / 2}$ (Ref. 25) with increasing separation between the monomers. Taking into account the ground-state dominance in the strong-randomness regime, we then estimate

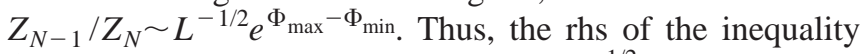
(38) overestimates the lhs by a factor of $L^{1 / 2}$, and the difference comes about precisely owing to the permutation signs in the expansion of $\operatorname{det} \hat{t}_{A-\alpha, B-\beta}$ [compare Eqs. (43) and (44)].

Finally, the defect pair energy for the optimized positions is simply $\mathfrak{e}_{\text {opt def }} / T_{d}=-\left(\Phi_{\max }-\Phi_{\min }\right)$, and we see that $Z_{N-1} / Z_{N} \sim L^{-1 / 2} \exp \left(-\mathfrak{e}_{\text {opt def }} / T_{d}\right)$; the factor $L^{-1 / 2}$ here represents the entropic cost for introducing two essentially fixed monomers into the dimer system.

Putting everything together, we may thus write $E_{\min }$ $\approx($ num factor $) \times L \exp \left(\mathfrak{e}_{\text {opt def }} / T_{d}\right)$. Thus, the dimer RG expression (23) underestimates the smallest positive energy by a factor $L^{-1}$ coming from the fermionic sign and dimer entropic origins.

Returning to the general case, we expect the intermediate bounds, as well as the dimer RG estimate from the optimized defects, to underestimate the smallest positive energy by some similar $L^{-r}$ factors. In our numerical tests (see below), we indeed find that the dimer RG provides a strong lower bound for $E_{\min }$. However, we cannot be more precise in the general case due to the following difficulty: Unlike the random vector potential case, we cannot disentangle the defect energetics from the dimer configurational combinatorics. More explicitly, the results of Ref. 12 suggest that a fixed pair of defects will have an average energy $O\left(+\ln \mid \mathbf{r}_{\alpha}\right.$ $-\mathbf{r}_{\beta} \mid$ ) because the system constrained by the presence of the defects cannot take as much advantage of the low-energy bonds, and $\mathfrak{e}_{\text {opt deft }}$ will have a similar $O(+\ln L)$ contribution in addition to the dominant $O\left(-(\ln L)^{3 / 2}\right)$ term coming from $v_{\min }-v_{\max }$.

\section{NUMERICAL TESTS}

We have performed extensive numerical tests using exact diagonalization and numerical RG. The results are consistent

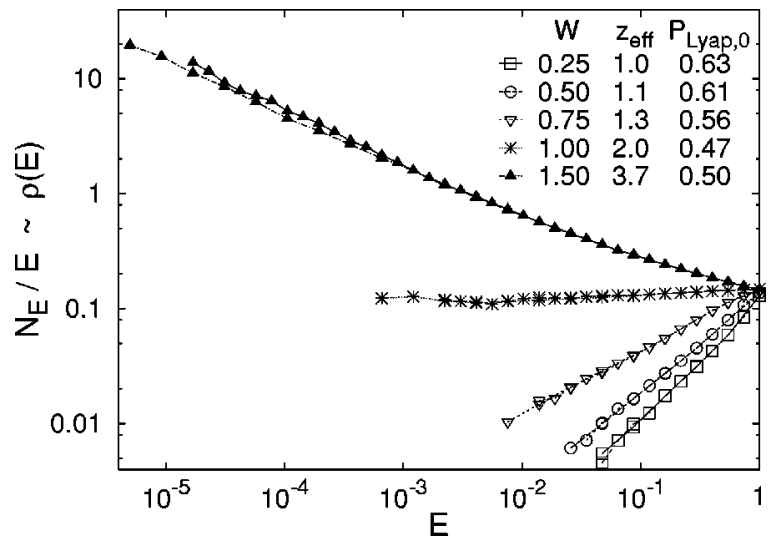

FIG. 4. Illustration of the "apparently nonuniversal" behavior of the density states (normalized per lattice site). The system is a $192 \times 192$ honeycomb lattice with hopping elements chosen randomly from a uniform distribution in $[1-W, 1+W]$; free boundary conditions are used in one direction and periodic bc in the other [see Fig. 5(a)]. The data are averaged over ten disorder realizations; the lowest energy shown in each case corresponds roughly to the second-lowest energy state in the finite sample. Similar data for a smaller $96 \times 96$ system is also shown; from such comparisons, we conclude that the density of states plotted is indeed the bulk density of states. The effective dynamical exponent $z_{\text {eff }}$ is obtained by fitting the integrated density of states $N_{E}$ to the form $N_{E} \sim E^{d / z}$ over the range $2<L^{2} N_{E}<200$ in each case. Direction-averaged Lyapunov spectrum density $\overline{\mathcal{P}}_{\text {Lyap }, 0}$ is calculated from numerical transfer matrix studies and provides a rough idea of the conducting properties of the system.

with the generalized Gade scaling Eq. (3) in the delocalized phase. Although direct diagonalization methods do not allow us to unambiguously distinguish the different exponents $x$ in the generalized Gade scaling, indirect methods provide strong indications in favor of $x=\frac{3}{2}$ different from the original Gade prediction of $x=2$. In the localized phase, the numerical results are more conclusive, and all methods clearly point to a power-law density of states (with nonuniversal dynamical exponent) generated by the "string" Griffiths mechanism discussed earlier.

\section{A. Illustration}

We first demonstrate some difficulties that plague direct diagonalization studies. In this context, it is important to note that a strong Gade-like divergence in the low-energy density of states has never been observed in previous numerical studies. ${ }^{27,28}$ In our direct diagonalization studies, we typically see power-law diverging or even vanishing density of states with nonuniversal exponents. This is illustrated in Fig. 4 where we show the calculated ${ }^{29,30}$ density of states $\rho(E)$ for the honeycomb lattice with hopping amplitudes chosen uniformly from $[1-W, 1+W]$. For $W<1$, on the length scales studied, the density of states is apparently power-law vanishing, with the observed effective exponent $z_{\text {eff }}$ that depends on the strength of the disorder $W$. It is simply impossible from the data to guess that there may be something else happening at still lower energy scales. For $W=1.0$ the density of states is almost constant, with a slight 
hint on some weak divergence at still lower energies observed for the largest system studied. For $W>1.0$ (the honeycomb lattice is still statistically isotropic in spite of the negative hopping amplitudes allowed, but the direct dimer connection is lost in this case), a power-law divergence is observed, and in this case, we also see some curvature in the $\log -\log$ plot of $\rho(E)$ vs $E$ suggestive of an even stronger divergence at still lower energy; however, from these numerical results it is not possible to say anything more about the nature of the true low-energy singularity in the DOS.

A partial resolution of this discrepancy between direct numerics and theory lies in the fact that the asymptotic regime, in which the form (2) is expected to hold, happens to be quite inaccessible for the exact diagonalization studies, given the computational restriction on the system size. Taking the original results of Ref. 1 at face value for the moment, we see that the asymptotic form is valid only below a crossover scale $^{31}$

$$
E_{\text {cross }} \sim \Omega_{0} e^{-16 \pi^{2} y^{2} \bar{\sigma}^{2}},
$$

where $\Omega_{0}$ is some bare energy, $\bar{\sigma}$ is the dimensionless conductivity of the 2D system, and $y$ is some dimensionless parameter that is greater than one. The conductivity $\bar{\sigma}$ that enters here can be estimated as $\bar{\sigma} \sim \mathcal{P}_{\text {Lyap }, 0}$ with some $O(1)$ prefactor; here $\mathcal{P}_{\text {Lyap }, 0}$ is the relevant Lyapunov spectrum density that can be obtained from a numerical transfer matrix analysis (see the following section). In Fig. 4, we have, therefore, also listed the corresponding values of $\mathcal{P}_{\text {Lyap }, 0}$ obtained for our system. The important thing to notice is that for the typically studied systems, the estimated $\bar{\sigma}$ is of order one and can vary somewhat. To get some feeling of the numbers involved, we set $y=1$ and check how $E_{\text {cross }}$ changes as we vary $\bar{\sigma}$. For the three values $\bar{\sigma}=1.0,0.5$, and 0.2 , we get $E_{\text {cross }} / \Omega_{0}=10^{-69}, 10^{-17}$, and $10^{-3}$, respectively. For energies above the crossover energy, Gade predicts an effective power-law density of states, and this is probably all that has been observed in the previous numerical studies (but see the very recent Ref. 32).

Furthermore, the modified Gade form (2) with $x=\frac{3}{2}$ proposed here has a weaker divergence than the earlier prediction of Gade. This is probably another reason why it has proved difficult to observe anything other than power-law singularities in the direct diagonalization studies. Of course, we expect the actual crossovers to be correspondingly more complicated, ${ }^{20}$ but estimates similar to the ones obtained from Gade's original work are still expected to hold. The upshot of all this is that one should therefore be very cautious while looking for the asymptotic behavior in two dimensions.

Finally, note that the foregoing observation suggests a way to bring the crossover energy up into the range accessible to direct numerical studies - the idea is to make the system look less delocalized, and this is what we turn to shortly.

\section{B. The choice of the system}

In the following, we present our numerical results only for one specific system-the brickwall (honeycomb) lattice of

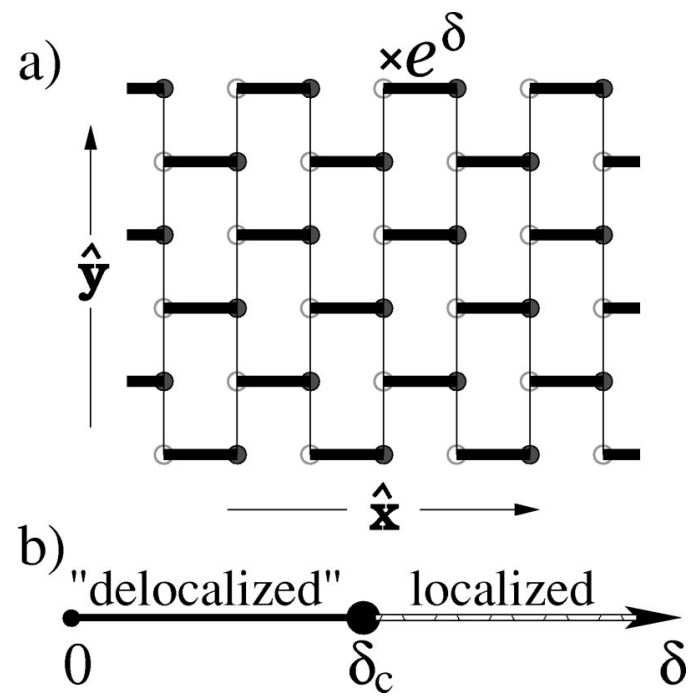

FIG. 5. (a) Brickwall lattice system we study numerically. Different groups of hopping amplitudes are chosen from different uniform distributions $[0, J]$, with $J=1$ for thin lines (regular bonds) and $J=e^{\delta}$ for dark thick lines (dimerized bonds). (b) Phase diagram of this system from transfer matrix analysis; $\delta_{c} \approx 1.432$.

Fig. 5(a). Nearest-neighbor hopping amplitudes $t_{e}$ are taken randomly and independently from uniform distribution $\left[0, J_{e}\right]$. When $J_{e} \equiv 1$ for all bonds of the lattice (i.e., all $t_{e}$ 's are taken from the same distribution and the underlying honeycomb lattice is statistically isotropic), the system is not exponentially localized at $E=0$, as can be checked by direct transfer matrix analysis. By allowing the horizontal bonds to be stronger on average, $J_{e}=e^{\delta}$ for the dark thick bonds in Fig. 5(a) (corresponding to an anisotropic honeycomb lattice), we can drive the system into a localized state for strong enough $\delta>\delta_{c}$, but the system remains "delocalized" for weak $\delta<\delta_{c}$ [see Fig. 5(b) for the phase diagram].

The rationale behind our specific choice of the system is as follows: We want to perform all numerics on the same system, so that we can compare results of different approaches. It is, therefore, best to use a system that has the precise connection $\operatorname{det} \hat{t}_{A B}=Z_{d}[A, B]$ with the corresponding random dimer problem, so that we can further check our arguments of Sec. IV B. Moreover, we want a system whose statistically isotropic version (i.e., without any enforced dimerization in values of the hopping amplitudes) shows an appreciable singularity in the low-energy DOS for accessible system sizes [this last requirement rules out the $\pi$-flux lattice-the largest $z_{\text {eff }}$ that we can observe in this system with order-one bare randomness is $\left.z_{\text {eff }}(L=192) \cong 1.5\right]$.

Of course, the fact that our lattice has the precise dimer connection does not mean that the results presented below are nongeneric. We also studied the honeycomb and square lattices with random bonds of any sign and obtained qualitatively similar results. In these systems with disorder strength of order one, we actually observe a stronger effective divergence in $\rho(E)$, which can be more readily checked against the precise functional form, but only through multiparameter fits, which are not entirely convincing. Since we can alternatively bring the crossover energy up into the accessible range 
by increasing $\delta$ and simultaneously preserving the dimer connection, this is what we choose to do, in part because the more precise "dimer" arguments provide us with the strongest evidence in favor of the proposed modified Gade scaling. However, we do expect that the suggestive strongrandomness association of the low-energy states in the hopping problem with the optimized defects in the dimer problem is likely still valid in some effective sense even when the fermionic problem does not have the precise $E$ $=0$ dimer connection.

\section{Transfer matrix analysis}

We now summarize the transfer matrix analysis that gives us the phase diagram Fig. 5(b), and also explain our procedure for estimating the conductivity $\bar{\sigma}$. The general setting is as follows: ${ }^{33,34}$ Consider transferring the wave function along a strip of transverse size $N \equiv L_{\perp}$. The transfer matrix is a $2 N \times 2 N$ matrix, and we calculate the Lyapunov spectrum of the corresponding random matrix product. The $2 N$ Lyapunov exponents $\nu_{i}$ come in \pm pairs, and we focus on the positive half of the Lyapunov spectrum $\nu_{1} \geqslant \cdots \geqslant \nu_{N} \geqslant 0$. The largest localization length $\xi_{\max }$ in the strip is obtained as the inverse of the smallest (in absolute value) Lyapunov exponent, $\xi_{\max }$ $\equiv 1 / \nu_{N}$. If $\xi_{\max }(N)$ is found to increase linearly with $N$, the system is said to be delocalized (more precisely, the 2D system is not exponentially localized). If, on the other hand, $\xi_{\max }(N \rightarrow \infty)$ saturates to a finite value, the 2D system is localized. Alternatively, ${ }^{33}$ the system is delocalized if there is a finite density of Lyapunov exponents

$$
\mathcal{P}_{\text {Lyap }}(\nu) \equiv \lim _{N \rightarrow \infty} N^{-1} \sum_{i} \delta\left(\nu-\nu_{i}\right)
$$

at $\nu=0$; similarly, the system is localized if there is a gap in the Lyapunov spectrum around $\nu=0$. Moreover, in the delocalized state, the Lyapunov spectrum density $\mathcal{P}_{\text {Lyap }, 0}$ $\equiv \mathcal{P}_{\text {Lyap }}(\nu=0)$ at $\nu=0$ gives us a measure of the conducting properties of the system.

In our bipartite hopping problem at $E=0$, we observe that the two sublattices decouple, and it is advantageous to transfer on one sublattice only (say, the $A$ sublattice), recovering the Lyapunov spectrum of the whole system from appropriate symmetries. Furthermore, for the brickwall lattice of Fig. 5 (a), the sublattice transfer in the $\hat{\mathbf{x}}$ direction involves only one column of $A$ sites at a time, but now the individual sublattice Lyapunov spectrum is not symmetric around zero. (In passing we note that in such a system with a free left boundary, there will be edge states living on the $A$ sites of the boundary, and the number of such edge states is precisely the number of negative Lyapunov exponents for the transfer in this case.) We employ all these simplifications in our numerical calculations; however, for the final results, we always quote the $\mathcal{P}_{\text {Lyap }, 0}$ for the whole system including both sublattices.

For the brickwall lattice of Fig. 5(a), there are two inequivalent directions $\hat{\mathbf{x}}$ and $\hat{\mathbf{y}}$. However, we have checked that the system is either delocalized or localized in both directions. We, therefore, expect the system to exhibit true $2 \mathrm{D}$

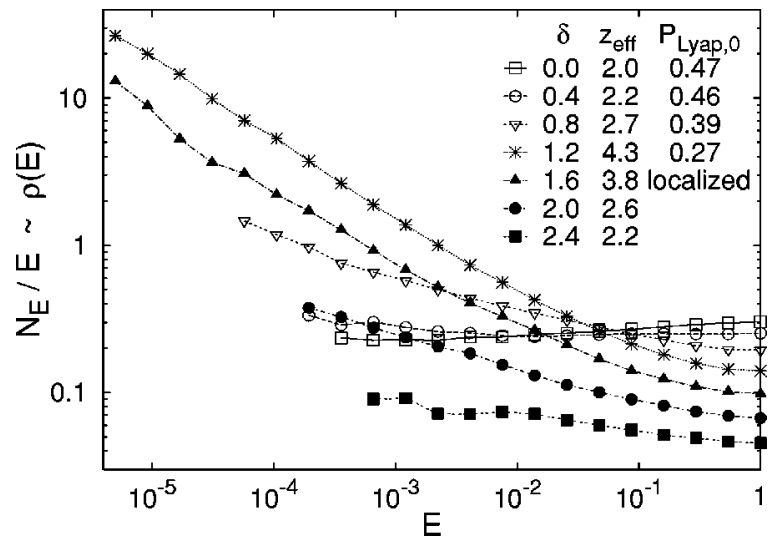

FIG. 6. Density of states for the anisotropic brickwall lattice of Fig. 5 calculated for $192 \times 192$ system with open bc in the $\hat{\mathbf{y}}$ direction and periodic bc in the $\hat{\mathbf{x}}$ direction. The regular (vertical) hopping amplitudes are chosen randomly from a uniform distribution in $[0,1]$, while the dimerized (horizontal) hopping amplitudes are chosen from $\left[0, e^{\delta}\right]$. The more detailed characterization of the data is similar to that of Fig. 4.

behavior in the delocalized phase as long as one is not too close to the localization transition. Our numerical results are given in the corresponding Figs. 4 and 6 . To estimate $\mathcal{P}_{\text {Lyap }, 0}$, we typically use several Lyapunov exponents that are closest in absolute value to zero, and we quote only the directionaveraged $\overline{\mathcal{P}}_{\text {Lyap }, 0} \equiv \sqrt{\mathcal{P}_{\text {Lyap }, 0}^{x x} \mathcal{P}_{\text {Lyap }, 0}^{y y}}$. (If the Lyapunov exponents are defined as growth exponents per unit length instead of per lattice unit, and if the corresponding density is also defined per transverse length, the lattice spacings $a_{x}$ and $a_{y}$ enter both $\mathcal{P}_{\text {Lyap }, 0}^{x x} \sim a_{x} / a_{y}$ and $\mathcal{P}_{\text {Lyap }, 0}^{y y} \sim a_{y} / a_{x}$, but not $\left.\overline{\mathcal{P}}_{\text {Lyap }, 0}.\right)$

We find that the system is indeed delocalized for $\delta=0$. The fact that it remains delocalized for some range of values $\delta>0$ can also be seen from the data at $\delta=0$ by considering the transfer on the $A$ sublattice in the $\hat{\mathbf{x}}$ direction: In this case, multiplying the horizontal bonds by $e^{\delta}$ simply shifts the whole Lyapunov spectrum rigidly down by $\delta, \nu_{i} \rightarrow \nu_{i}-\delta$. Thus, there remains some finite Lyapunov spectrum density $\mathcal{P}_{\text {Lyap }, 0}$ for a range of $\delta$, until the top of the spectrum reaches $\nu=0$, and only then does the system localize. The critical $\delta_{c}$ can be estimated very accurately, since we need to know only the largest Lyapunov exponent in the $\delta=0$ case; for the particular system studied, we find $\delta_{c} \approx 1.432$.

What "conducting" property of the system does the finite $\mathcal{P}_{\text {Lyap, } 0}$ correspond to? Chalker and Bernhard ${ }^{33}$ suggest that $\mathcal{P}_{\text {Lyap, } 0}$ gives, up to some numerical factor, the conductivity of the 2D system in units of $e^{2} / h$,

$$
\bar{\sigma} \sim \mathcal{P}_{\text {Lyap }, 0} .
$$

However, we have to ask ourselves which conductivity is actually being "measured" by Eq. (47), particularly since we are dealing with systems that have a singular DOS at the Fermi level. In this respect, note that the above transfer matrix approach can be equivalently formulated as a recursion procedure for calculating the Green's function $(E-\hat{H})^{-1}$ between the first and the last slices of the long strip, ${ }^{34}$ while the 
largest localization length from such transfer matrix analysis corresponds to the average modulus squared of such a "propagator" between the two slices. This transfer matrix estimate resembles the definition of conductivity used in the sigma-model literature (see, e.g., Refs. 4 and 35), which is the conductivity we need in Eq. (45). To make our estimates more consistent, we fix the proportionality factor in Eq. (47) by using the exact results for some pure systems. Thus, an elementary calculation for the pure $\pi$-flux model gives $\mathcal{P}_{\text {Lyap }, 0}=2 / \pi$, which should be compared with $\bar{\sigma}=2(1 / \pi)$ for the two Dirac points of the model's continuum limit (see Ref. 4). From this and similar considerations for the brickwall lattice ${ }^{36}$ we conclude that the accurate relationship is simply $\bar{\sigma}=\mathcal{P}_{\text {Lyap }, 0}$.

Finally, we remark a couple of asides. The first concerns the random vector potential model, for which Ref. 4 predicts $\bar{\sigma}=2 / \pi$ for any strength of the randomness. This result can be also obtained within the above transfer matrix approach, if we assume that we can indeed fix the unknown numerical factor in Eq. (45) to the appropriate constant value. When we add the random surface $\Phi(\mathbf{r})$ on top of, say, the pure $\pi$-flux model [see Eq. (14)], the $E=0$ transfered wave functions need to be simply multiplied by the appropriate $e^{-\Phi(\alpha)}$ or $e^{\Phi(\beta)}$. But this cannot change the Lyapunov spectrum of the transfer along the quasi-1D strip for any finite transverse size of the strip, because $\Phi(\mathbf{r})$ in such geometry is effectively a one-dimensional Gaussian surface and cannot fluctuate stronger than $\left[\Phi\left(L_{\|}\right)-\Phi(0)\right]^{2} \sim L_{\|}$along the strip. At this stage, it is not clear to us whether $\bar{\sigma}$ should have any signature of the "freezing" transition at $g_{c}$ observed in the static and dynamic properties of this system; the above argument seems to suggest that there is none, but we cannot rule out the possibility that taking the limit $L_{\|} \rightarrow \infty$ while keeping $L_{\perp}$ fixed "loses" the information about the 2D system.

The second remark concerns the brickwall lattice of Fig. 5 (a) and the transfer in the $\hat{\mathbf{x}}$ direction. In this case, there is a direct correspondence between the conducting properties of the system as measured by the Lyapunov spectrum and the energetics of the domain walls in the corresponding dimer problem. Such domain walls are defined relative to the fully locked state that is obtained for $\delta>\delta_{c}$, and are forced to run in the $\hat{\mathbf{x}}$ direction and cannot terminate; their number at a given $\delta$ is precisely the number of positive Lyapunov exponents in the hopping problem, while the free energy cost per unit length of adding or removing domain walls is precisely the spacing between the Lyapunov exponents near $\nu=0$.

\section{Exact diagonalization studies: Density of states}

We also monitor the density of states as we increase the dimerization from $\delta=0$, eventually driving the system into a localized state for $\delta>\delta_{c}$. The corresponding results are shown in Fig. 6, together with the information about the system's localization properties. Consistent with our earlier discussion, the DOS divergence is seen to become stronger as we approach the localization transition, with the effective exponent $z_{\text {eff }}(L=192)$ peaking at the transition. Deep in the localized phase, the log-log plot of $\rho(E)$ vs $E$ is indeed a straight line, and the quoted values of $z$ give the actual bulk dynamical exponents. In the delocalized phase, on the other hand, there is still some curvature at the lowest energy scale, and the extracted values $z_{\text {eff }}$ can only serve as rough indicators of the strength of the divergence.

The actual data analysis is performed as follows: In the delocalized phase, we fit the integrated density of states $N_{E}$ to the generalized Gade form

$$
N_{E}=a \Gamma_{E}^{1-1 / x} e^{-c \Gamma_{E}^{1 / x}}
$$

where $\Gamma_{E} \equiv \ln \left(\Omega_{0} / E\right)$. Specifically, we try two values for the exponent $x$-the original Gade $x=2$ and the modified $x$ $=\frac{3}{2}$. The included prefactor $\Gamma_{E}^{1-1 / x}$ is based on an assumption that there are no additional corrections to the asymptotic $E \rightarrow 0$ form $\rho(E) \sim E^{-1} \exp \left(-\Gamma_{E}^{1 / x}\right)$. This is something that we do not really know-in fact, our lowest-gap analysis of Sec. IV B seems to suggest that there may be corrections stronger than any such $\Gamma^{r}$ prefactor. In this situation, we treat the above fit function only as a baseline. To be consistent, we should also allow for the uncertainty $\Gamma_{E} \rightarrow \Gamma_{E}+\Gamma_{0}$ in the bare energy scale relative to which the $\log$ scale $\Gamma$ is defined. As one might suspect, for a fixed fit range, we can approximate any DOS curve with the three parameters $a, c$, and $\Gamma_{0}$, so this sort of analysis is not really conclusive. What we can do, however, is to look for the overall consistency of such fits: We can ask, for example, how the fit parameters change as we change the fit region, or how sensible the obtained parameters are (thus, the parameter $a$ should be of order one). Generally, the $x=\frac{3}{2}$ form fares somewhat better in such analysis; also, the extracted numerical value of the parameter $c$ from such fits compares favorably with the lowest-gap results of the following section. But this is as far as our direct DOS studies can take us in the delocalized phase.

In the localized phase, we have also tried fitting the DOS using a power-law times a logarithmic correction $N_{E}$ $\sim E^{d / z}\left(\Gamma_{E}\right)^{r}$. We typically find that the best fit corresponds to $r \cong 0$, suggesting that the density of states has a simple power-law form in the localized phase. This is also our conclusion from the lowest-gap studies presented below.

\section{E. The lowest-energy state: Exact diagonalization and dimer optimized defects studies}

We can extend our numerical tests further by considering the distribution of the smallest positive energy in finite samples, paying particular attention to the scaling with sample size. Such a direct diagonalization study can be performed for a factor of 2 larger systems than in our DOS studies, while indirect dimer methods can take us another factor of 4 in system size. This well-controlled and rather sensitive numerical approach also corresponds closely to our analytical arguments in Sec. IV B.

We consider the same brickwall lattice system of Fig. $5(\mathrm{a})$; however, we now consider $\left(2 M_{x}+1\right) \times 2 M_{y}$ "odd $\times$ even" samples with spiral boundary conditions in the $\hat{\mathbf{x}}$ direction and free boundaries in the $\hat{\mathbf{y}}$ direction. The specific choice of the boundary conditions is such that the dimer equivalence $\operatorname{det} \hat{t}_{A B}=Z_{d}[A, B]$ holds precisely for this 


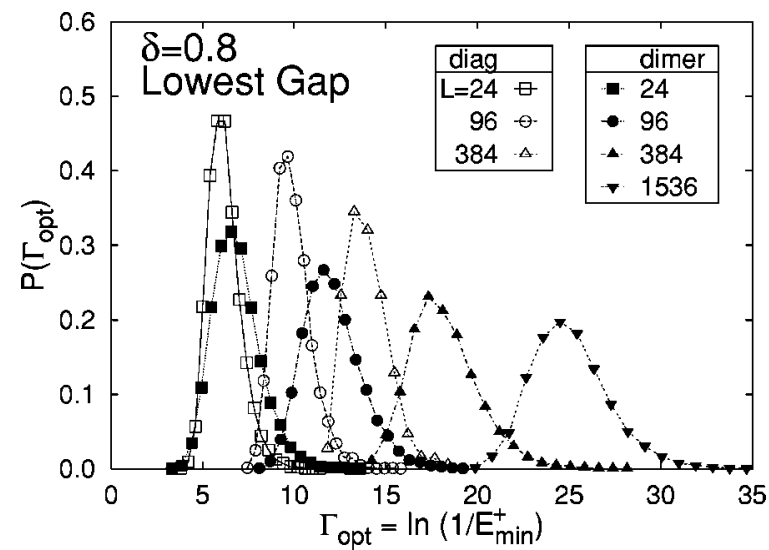

FIG. 7. "Lowest-gap" analysis in the delocalized phase $(\delta$ $=0.8$ ) of the system of Fig. 5. We consider $(L+1) \times L$ finite samples with spiral bc in the $\hat{\mathbf{x}}$ direction and free $b c$ in the $\hat{\mathbf{y}}$ direction. Open symbols show distributions of the smallest positive energy calculated by exact diagonalization methods, while filled symbols show distributions of the corresponding dimer RG "estimate" Eq. (23).

system $;{ }^{37}$ this allows us to compare the lowest-energy state of the hopping problem with the optimized defects in the corresponding dimer problem, exactly paralleling the discussion of Sec. IV B. To this end, we also calculate ${ }^{38,39}$ the dimer RG "estimate" Eq. (23) for the lowest gap, which is the quantity used in the bounds of Sec. IV B.

For the purposes of illustration, we show our results for two values of $\delta$, one in the delocalized phase $(\delta=0.8)$ and one in the localized phase $(\delta=2.0)$, both chosen well away from the transition point $\left(\delta_{c}=1.432\right)$.

\section{Delocalized phase, $\delta=0.8$}

Figure 7 shows distributions of the logarithm of the smallest positive energy in our samples in the delocalized phase. The exact diagonalization results for $\Gamma_{\mathrm{opt}}=\ln \left(1 / E_{\min }^{+}\right)$are shown with open symbols and extend up to system sizes $L$ $=384$; while the corresponding dimer $\mathrm{RG}$ estimates $-\mathfrak{e}_{\text {opt def }}$ [cf. Eq. (23)] are shown with filled symbols and extend up to $L=1536$. We see that the distributions move out strongly to larger absolute log-energies (i.e., smaller energies) with increasing $L$ and broaden somewhat at the same time. We also see that the dimer RG estimates for the lowest gap are consistently smaller and indeed provide strong lower bounds, at least for the larger systems. This is in agreement with our discussion in Sec. IV B.

In Fig. 8, we analyze the behavior of the distributions $P\left(\Gamma_{\text {opt }} ; L\right)$ of Fig. 7 , plotting their mean $\overline{\Gamma_{\text {opt }}}(L)$ and the standard deviation $\sigma\left(\Gamma_{\text {opt }} ; L\right)$. On a plot with linear scales for both $\ln L$ and $\Gamma_{\text {opt }}$ (not shown), we observe a visible curvature $\overline{\Gamma_{\mathrm{opt}}} \sim(\ln L)^{x}$ with $x>1$, indicative of a stronger than any finite- $z$ singularity. In Fig. 8, we use logarithmic scales for both $\ln L$ and $\Gamma_{\text {opt }}$ to get a rough estimate of the exponent $x$. The exact diagonalization results are seen to fall between the $x=1$ and $x=\frac{3}{2}$ lines, well away from the Gade $x=2$; at the same time, the optimized defect results, which extend to larger system sizes, clearly approach the $x=\frac{3}{2}$ line,

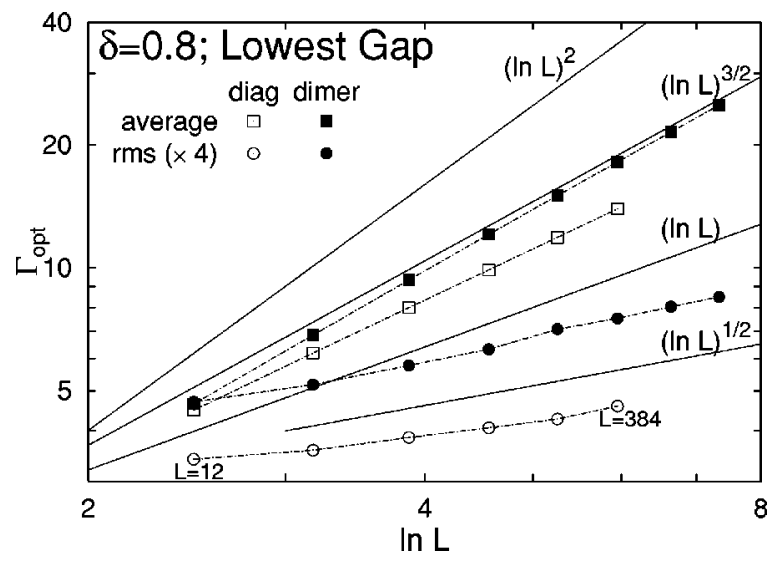

FIG. 8. Detailed analysis of the distributions of Fig. 7. The mean $\overline{\Gamma_{\text {opt }}}(L)$ and the standard deviation $\sigma\left(\Gamma_{\text {opt }} ; L\right)$ are plotted with open and filled symbols for the exact diagonalization and dimer RG results correspondingly. Note the logarithmic scale for both the $\ln L$ and $\Gamma_{\text {opt }}$ axes. To gauge the $L$ dependence of $\overline{\Gamma_{\text {opt }}}$ and $\sigma\left(\Gamma_{\text {opt }}\right)$, we also plot different powers $(\ln L)^{x}$ with $x=2, \frac{3}{2}, 1$, and $\frac{1}{2}$.

as has been already checked in Ref. 12 for a related model. We also note a much weaker $\sim(\ln L)^{1 / 2}$ dependence of the width of the distributions on the system size, consistent with the results of Ref. 12 (summarized in Sec. IV B).

In a more quantitative analysis, following Ref. 12, we perform linear fits for $\left[\overline{\Gamma_{\text {opt }}}\right]^{2 / 3}$ and $\left[\sigma\left(\Gamma_{\text {opt }}\right)\right]^{2}$ as functions of $\ln L$. For the exact diagonalization data, we obtain the asymptotic scaling $\overline{\Gamma_{\text {opt }}} \approx 0.81(\ln L)^{3 / 2}$ and $\sigma \approx 0.42(\ln L)^{1 / 2}$. We can compare this with the result from the DOS studies: performing the generalized Gade fit Eq. (48) of the data of Fig. 6, we extract the parameter $c \cong 2.2$, which translates to a fairly close asymptotic scaling prediction $\overline{\Gamma_{\mathrm{opt}}}$ $\approx 0.87(\ln L)^{3 / 2}$. Similarly, the dimer $\mathrm{RG}$ data yields $\overline{\Gamma_{\text {opt }}}$ $\approx 1.30(\ln L)^{3 / 2}$, not unreasonably far from the above exact diagonalization predictions [as discussed in Sec. IV B, we expect stronger subleading $O(\ln L)$ terms for the exact lowest gap $\Gamma_{\text {opt }}$ than for the dimer $\left.-\mathfrak{e}_{\text {opt def }}\right]$. Finally, we note that Ref. 40 calculated the bulk defect density in a related vortex glass model as a function of the defect core energy (which corresponds to $\Gamma_{\text {opt }}$ in the fermion problem), obtaining $N_{\Gamma} \sim \exp \left(-c \Gamma^{0.74}\right)$, fairly close to the expected $N_{\Gamma}$ $\sim \exp \left(-c \Gamma^{1 / x}\right)$ scaling form with $x=\frac{3}{2}$.

\section{Localized phase, $\delta=2.0$}

The lowest-gap analysis is simpler in the localized phase, and is shown in Figs. 9 and 10. In this case too, the distributions move out to larger $\Gamma_{\text {opt }}$ with increasing $L$, but now do so at a slower pace. We clearly observe simple finite- $z$ scaling $\overline{\Gamma_{\text {opt }}}=$ const $+z \ln L$, with $z=2.56$, which compares well with the estimate of $z=2.64$ from the DOS studies. Also, the widths of the distributions essentially do not change with the system size. Note that the effects discussed in Sec. IV B produce a much smaller difference between the exact diagonalization results and the dimer RG estimates in the localized phase, since the distance between the optimal defects is expected to scale very weakly $\sim \ln L$ with the sample size. Thus, at least at $\delta=2.0$ deep in the localized phase, and for 


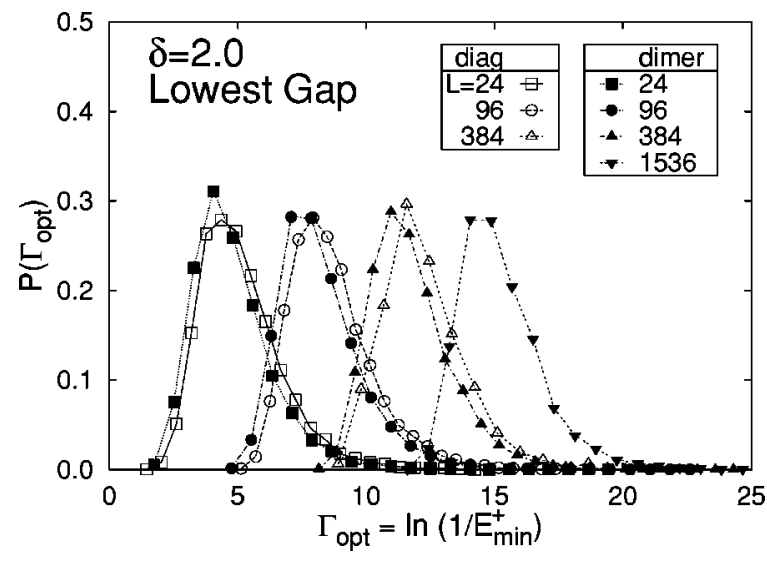

FIG. 9. Lowest-gap analysis in the localized phase, $\delta=2.0$, similar to that of Fig. 7.

the sizes studied, the dimer RG gives numerically accurate results for the lowest gap and the two peaks of the corresponding wave function in each sample.

\section{DISCUSSION}

Our main results have already been summarized in the Introduction, and here, we confine ourselves to some unresolved questions in these bipartite models, as well as some issues in the closely related ImRH problems.

Our first remark concerns the zero-energy wave function in the "delocalized" critical phase of the general bipartite problem. The multifractal properties of this wave function are expected to be trivial $\tau(q) \equiv 0$ - this is due to the flow to strong disorder $g \rightarrow \infty$ (see, e.g., Ref. 10). Equivalently, since the logarithm of the wave function magnitude is essentially the potential $v(\mathbf{r})$ seen by the defects of the corresponding dimer problem [see Eq. (39)], none of the participation ratios depends on system size-this is a consequence of the ground-state dominance for this random potential. In this respect, the critical wave function looks fairly localized. The typical correlations such as $C_{\text {typ }}(r)=\exp (\overline{\ln |\Psi(r) \Psi(0)|})$, are thus directly related to the correlations of $v(\mathbf{r})$. On the other hand, the situation is less clear when we consider average correlations such as $C_{\text {av }}(r)=\overline{|\Psi(\mathbf{r}) \Psi(0)|}$. Estimating

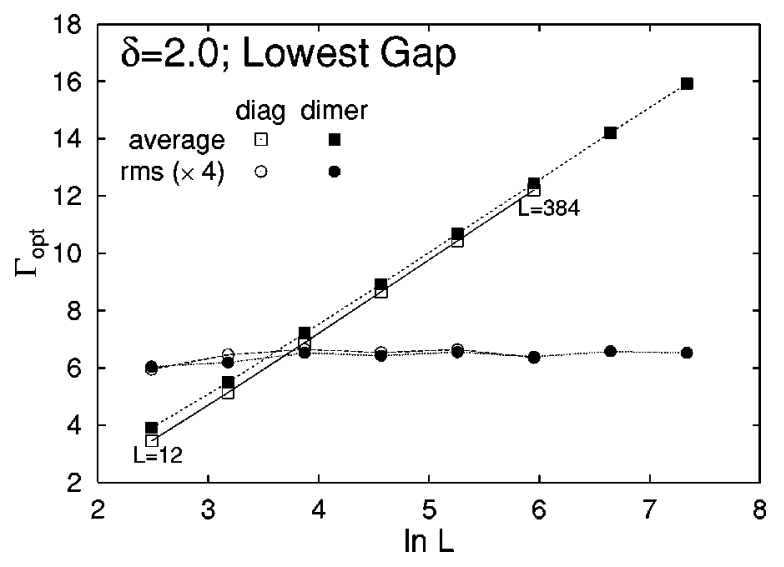

FIG. 10. Analysis of the distributions of Fig. 9. these requires a better understanding of the statistics of such random surfaces. For instance, it is not clear if $C_{\mathrm{av}}(r)$ is dominated by the near returns of the surface to its global extrema, as is the case in the one-dimensional chain. Moreover, if this is indeed the case, what is the probability of finding such quasidegenerate minima at a fixed distance $r$ ?

Another feature of the delocalized phase that we have not discussed is the suggested ${ }^{9,41}$ universal susceptibility variations in the vortex glass problem, and it would be interesting to study the corresponding response in the fermionic problem in more detail.

The character of the transition between the delocalized and localized phases is a completely separate issue that we have ignored altogether. It is not obvious if this critical end point of the line of fixed points that characterize the metallic phase exhibits dynamical scaling distinct from the metallic phase. Another interesting feature of this transition is that the conductivity $\bar{\sigma}_{x x}$ remains finite (because the Lyapunov spectrum density is finite at the top of the Lyapunov band), while $\bar{\sigma}_{y y}$ vanishes. It is not clear how this extreme anisotropy affects the properties of the transition, and whether anything interesting remains.

In the localized phase, we have not discussed in detail the structure of our low-energy "string" excitations. Presumably, they look similar to the domain walls (flux lines) in the corresponding random dimer (vortex glass) problem. Note that in the particular localized phase that we studied, the strings are forced to run in the $\hat{\mathbf{x}}$ direction. In a more general localized phase, obtained by introducing some other dimerization pattern, the situation is more complicated, but it still seems that it will be some kind of directed strings that will contribute most to the low-energy density of states: The strings need to be stretched so that the end-to-end distance (setting the tunneling frequency scale) is of order the string length (determining the occurrence probability) for our counting arguments of Sec. III B to work. An interesting system to study in this respect is a 2D spinless superconductor with timereversal invariance, which maps onto a bilayer BPRH. When the " $p_{x}$-wave" superconducting pairing amplitude $\Delta_{\mathbf{j}, \mathbf{j}+\mathbf{x}}$ is zero, the system is just a doubled Anderson localization problem, whereas increasing $\Delta$ from zero, we obtain a bipartite localized phase "connected" to the standard Anderson insulator point. Note the richness of the full phase diagram in this system: a simple Lyapunov spectrum shift argument shows that increasing $\Delta$ further drives the system from this localized phase first into a critical delocalized phase, and then again into a localized phase similar to our staggered band insulator.

We conclude with some comments regarding more generic ImRH problems. As is clear from the preceding discussion, we expect similar Griffiths-string effects in the localized phases of such systems. An important question is, of course, whether this Griffiths mechanism can compete with other mechanisms of "filling the gap" (which can, for example, produce a constant contribution to the density of states at the band center as in Ref. 5). In particular, are there situations with a power-law divergent density of states that has its origins in such Griffiths effects? While we have not studied these questions in detail, there are certainly situations 
where this does indeed happen. Thus, in the particular fermionic ImRH representation ${ }^{42}$ of the two-dimensional random bond Ising model, our preliminary results suggest that the density of states is power-law vanishing to the left (the lessdisordered side) of the Nishimori line and power-law diverging to the right (this particular example was suggested to us by Nick Read and the corresponding result proved for a onedimensional toy model in Ref. 43). Finally, 2D ImRH systems are believed to also have true metallic phases, ${ }^{5}$ and it would be interesting to consider these from this perspective. In particular it remains to be seen whether one can use an analogous dimer connection to gain further insight into their properties.

Note added. Identical results for the dynamical freezing in the random vector potential model were also obtained independently in Ref. 44.

\section{ACKNOWLEDGMENTS}

We would like to thank N. Read for a useful discussion. The work at Princeton was supported by NSF grant No. DMR-9802468, and at Harvard by NSF Grant No. DMR9981283.

\section{APPENDIX: 2D GAUSSIAN SURFACE}

The following facts are used in the main text to obtain bounds for the dynamical scaling in the random vector potential model; these are transcribed directly from Ref. 11 for the two-dimensional Gaussian surface $\Phi(\mathbf{r})$, Eq. (15), in an $L \times L$ box [stipulating $\left.\int d \mathbf{r} \Phi(\mathbf{r})=0\right]$.
Extremal properties of the surface $\Phi(\mathbf{r})$ are sharply defined: e.g., the maximum scales as

$$
\Phi_{\max }(L)=2 \sqrt{(g / 2 \pi)} \ln L-\frac{3}{2} \sqrt{(g / 8 \pi)} \ln \ln L+\delta \Phi_{\max },
$$

with the sample-to-sample dependence entering only through an $O(1) \times \sqrt{g}$ random variable $\delta \Phi_{\max }$.

The "partition function"

$$
\mathfrak{Z}=\sum_{\mathbf{r}} e^{-2 \Phi(\mathbf{r})}
$$

has a sharply defined logarithm,

$$
\begin{array}{ll}
\ln \mathfrak{Z}_{g}(L)=2\left(1+\frac{g}{g_{c}}\right) \ln L+\Delta_{g}, & g<g_{c} \equiv 2 \pi ; \\
\ln \mathfrak{Z}_{g}(L)=4 \ln L-\frac{1}{2} \ln \ln L+\Delta_{g}, & g=g_{c} ; \\
\ln \mathfrak{Z}_{g}(L)=\sqrt{\left(g / g_{c}\right)}\left(4 \ln L-\frac{3}{2} \ln \ln L\right)+\Delta_{g}, & g>_{c} ;
\end{array}
$$

where $\Delta_{g}$ is an $O(1)$ random number (different in each case). Note that for strong disorder $g>g_{c}, \mathfrak{Z}(L)$ is dominated by the global minimum:

$$
\mathfrak{Z}_{g}(L)=e^{-2 \Phi_{\min }(L)} \sum_{\mathbf{r}} e^{-2\left[\Phi(\mathbf{r})-\Phi_{\min }(L)\right]},
$$

where the sum contributes no $L$ dependence because $\exp \left\{-2\left[\Phi(\mathbf{r})-\Phi_{\min }\right]\right\}$ remains normalizable in the limit $L \rightarrow \infty$.
${ }^{1}$ R. Gade, Nucl. Phys. B 398, 499 (1993).

${ }^{2}$ R. Gade and F. Wegner, Nucl. Phys. B 360, 213 (1991).

${ }^{3}$ S. Hikami, M. Shirai, and F. Wegner, Nucl. Phys. B 408, 415 (1993).

${ }^{4}$ A. W. W. Ludwig, M. P. A. Fisher, R. Shankar, and G. Grinstein, Phys. Rev. B 50, 7526 (1994).

${ }^{5}$ T. Senthil and M. P. A. Fisher, Phys. Rev. B 61, 9690 (2000).

${ }^{6}$ S. Guruswamy, A. LeClair, and A. W. W. Ludwig, Nucl. Phys. B 583, 475 (2000).

${ }^{7}$ J. L. Cardy and S. Ostlund, Phys. Rev. B 25, 6899 (1982).

${ }^{8}$ J. Toner and D. P. DiVincenzo, Phys. Rev. B 41, 632 (1990).

${ }^{9}$ T. Hwa and D. S. Fisher, Phys. Rev. Lett. 72, 2466 (1994).

${ }^{10}$ H. E. Castillo et al., Phys. Rev. B 56, 10668 (1997).

${ }^{11}$ D. Carpentier and P. Le Doussal, Phys. Rev. E 63, 026110 (2001).

${ }^{12}$ C. Zeng, P. L. Leath, and D. S. Fisher, Phys. Rev. Lett. 82, 1935 (1999).

${ }^{13}$ O. Motrunich, K. Damle, and D. A. Huse, Phys. Rev. B 63, 224204 (2001).

${ }^{14}$ M. R. Zirnbauer, J. Math. Phys. 37, 4986 (1996).

${ }^{15}$ Y. Hatsugai, X.-G. Wen, and M. Kohmoto, Phys. Rev. B 56, 1061 (1997).

${ }^{16}$ F. D. M. Haldane, Phys. Rev. Lett. 61, 2015 (1988).

${ }^{17}$ P. W. Kasteleyn, in Graph Theory and Theoretical Physics, edited by F. Harary (Academic, New York, 1967), pp. 44-110.
${ }^{18}$ E. W. Montroll, in Applied Combinatorial Mathematics, edited by E. F. Beckenbach (Wiley, New York, 1964), pp. 96-143.

${ }^{19}$ O. Motrunich, K. Damle, and D. A. Huse, Phys. Rev. B 63, 134424 (2001).

${ }^{20}$ P. Le Doussal and T. Giamarchi, Physica C 331, 233 (2000).

${ }^{21}$ P. W. Brouwer, C. Mudry, B. D. Simons, and A. Altland, Phys. Rev. Lett. 81, 862 (1998).

${ }^{22}$ P. W. Brouwer, C. Mudry, and A. Furusaki, Phys. Rev. Lett. 84, 2913 (2000).

${ }^{23}$ If a given lattice does not allow complete matchings, as can happen, e.g., when the numbers of $A$ and $B$ sites are not equal, or when there are some disconnected or "dangling" lattice parts, the system will have exact zero-energy states, and the number of such "graph zeros" is equal to the minimal number of free sites that are left uncovered by any partial dimer cover of the system (also called "deficiency" number of the bipartite graph).

${ }^{24}$ The leftmost inequality in Eq. (25) implies that the zero-energy RG Eq. (21) is always an upper bound for $E_{\min }^{+}$.

${ }^{25}$ M. E. Fisher and J. Stephenson, Phys. Rev. 132, 1411 (1963).

${ }^{26}$ H. E. Castillo and P. Le Doussal, cond-mat/0006373 (unpublished).

${ }^{27}$ S. N. Evangelou, J. Phys. C 19, 4291 (1986).

${ }^{28}$ Y. Morita and Y. Hatsugai, Phys. Rev. Lett. 79, 3728 (1997).

${ }^{29}$ Our numerical methods for both the DOS and the lowest gap 
employ crucially the bandedness of the corresponding matrix diagonalization problems (therefore free bc in one direction) and are based on routines from R. S. Martin and J. H. Wilkinson, in Handbook for Automatic Computation, edited by J. H. Wilkinson and C. Reinsch (Springer-Verlag, New York, 1971), Vol. II, Chap. 1.6, pp. $70-92$.

${ }^{30}$ The brickwall RH problem [Fig. 5(a)] with free bc in the $\hat{\mathbf{x}}$ direction has of order $L_{y}$ edge states with exponentially small (in $L_{x}$ ) energies living on the free $A$ and $B$ sites of the corresponding left and right boundaries. To study the bulk DOS, one would then have to separate the $O(L)$ edge and $O\left(L^{2}\right)$ bulk states in the total low-energy DOS, e.g., by varying the system size $L$. However, the bulk DOS can also be obtained more easily by having no free boundaries in the $\hat{\mathbf{x}}$ direction, and this is what we do throughout.

${ }^{31}$ Formula (45) is taken verbatim from Ref. 1, correcting for a minor inconsistency between Eqs. (6.6) and (6.12) there.

${ }^{32}$ S. Ryu and Y. Hatsugai, cond-mat/0107516 (unpublished).

${ }^{33}$ J. T. Chalker and M. Bernhardt, Phys. Rev. Lett. 70, 982 (1993).

${ }^{34}$ A. MacKinnon and B. Kramer, Z. Phys. B: Condens. Matter 53, 1 (1983)

${ }^{35}$ A. J. McKane and M. Stone, Ann. Phys. (N.Y.) 131, 36 (1981).

${ }^{36}$ Consider the staggered square lattice system defined as $t_{\mathbf{j}, \mathbf{j}}+\hat{\mathbf{x}}$ $=t_{x}+(-1)^{j_{x}+j_{y}} \Delta_{x}$ and $t_{\mathbf{j}, \mathbf{j}+\hat{\mathbf{y}}}=t_{y}$, which for $t_{x}<t_{y}$ has two Dirac points, with $v_{x}=2 a_{x} \Delta_{x}$ and $v_{y}=2 a_{y} \sqrt{t_{y}^{2}-t_{x}^{2}}$ [the brickwall lattice of Fig. 5(a) is a particular example with $\left.\Delta_{x} \equiv t_{x}\right]$. For the transfer in the two directions we obtain $\mathcal{P}_{\text {Lyap }, 0}^{x x}=\left(v_{x} / v_{y}\right)$ $(2 / \pi)$ and $\mathcal{P}_{\text {Lyap }, 0}^{y y}=\left(v_{y} / v_{x}\right)(2 / \pi)$ consistent with the anisotropic rescaling of the conductivity for the Dirac fermions in 2D.

${ }^{37} Z_{d}[A, B]=\operatorname{det} \hat{t}_{A B}$ for "odd $\times$ even" brickwall lattice with spiral bc in the $\hat{\mathbf{x}}$ direction and free bc in the $\hat{\mathbf{y}}$ direction because any transition cycle relative to the natural (horizontal) reference dimer cover winds precisely once along the $\hat{\mathbf{x}}$ direction and, therefore, has an odd number of $A \rightarrow B$ steps.

${ }^{38}$ A. A. Middleton, Phys. Rev. B 61, 14787 (2000).

${ }^{39}$ To find the optimized defects, we use a variant of the "maximumweight bipartite matching — shortest path" combination of Ref. 38.

${ }^{40}$ C. Zeng and P. L. Leath, Comput. Phys. Commun. 122, 531 (1999).

${ }^{41}$ C. Zeng, P. L. Leath, and T. Hwa, Phys. Rev. Lett. 83, 4860 (1999).

${ }^{42}$ J. A. Blackman and J. Poulter, Phys. Rev. B 44, 4374 (1991).

${ }^{43}$ I. A. Gruzberg, N. Read, and A. W. W. Ludwig, Phys. Rev. B 63, 104422 (2001).

${ }^{44}$ B. Horovitz and P. Le Doussal, cond-mat/0108143 (unpublished). 